\title{
Simulations of $P-S V$ wave scattering due to cracks by the 2-D finite difference method
}

\author{
Yuji Suzuki $^{1 *}$, Takahiro Shiina ${ }^{2 \dagger}$, Jun Kawahara ${ }^{3}$, Taro Okamoto ${ }^{4}$, and Kaoru Miyashita ${ }^{3}$ \\ ${ }^{1}$ Graduate School of Science and Engineering, Ibaraki University, Mito 310-8512, Japan \\ ${ }^{2}$ Research Center for Prediction of Earthquakes and Volcanic Eruptions, Graduate School of Science, \\ Tohoku University, Sendai 980-8578, Japan \\ ${ }^{3}$ Faculty of Science, Ibaraki University, Mito 310-8512, Japan \\ ${ }^{4}$ Department of Earth and Planetary Sciences, Tokyo Institute of Technology, Tokyo 152-8551, Japan
}

(Received September 3, 2012; Revised June 20, 2013; Accepted June 25, 2013; Online published December 6, 2013)

We simulate $P-S V$ wave scattering by 2-D parallel cracks using the finite difference method (FDM). Here, special emphasis is put on simplicity; we apply a standard FDM (second-order velocity-stress scheme with a staggered grid) to media including traction-free, infinitesimally thin cracks, which are expressed in a simple manner. As an accuracy test of the present method, we calculate the displacement discontinuity along an isolated crack caused by harmonic waves using the method, which is compared with the corresponding results based on a reliable boundary integral equation method. The test resultantly indicates that the present method yields sufficient accuracy. As an application of this method, we also simulate wave propagation in media with randomly distributed cracks. We experimentally determine the attenuation and velocity dispersion induced by scattering from the synthetic seismograms, using a waveform averaging technique. It is shown that the results are well explained by a theory based on the Foldy approximation, if the crack density is sufficiently low. The theory appears valid with a crack density up to at least 0.1 for $S V$ wave incidence, whereas the validity limit appears lower for $P$ wave incidence.

Key words: Scattering, cracked media, finite difference methods, attenuation, dispersion.

\section{Introduction}

Random inhomogeneities inside the Earth's lithosphere scatter high-frequency seismic waves and thereby cause phenomena such as the attenuation, dispersion, and generation of coda waves (Sato et al., 2012). The modeling of such inhomogeneities can be categorized into two concepts: random spatial fluctuation of medium parameters (called random media), and discrete scatterers such as cracks, inclusions, or cavities. Although the concept of random media has been popularly adopted in theoretically modeling seismic scattering (Sato et al., 2012), it assumes quite often a continuous heterogeneity. In contrast, the real Earth's interior obviously has a discontinuous nature. It includes a large amount of cracks or fractures, and it also has many irregular interfaces having a sharp material contrast. Such a discontinuous heterogeneity can be modeled using the concept of discrete scatterers, either directly or approximately (Benites et al., 1992).

One of the popular numerical methods to simulate seismic scattering due to discrete scatterers is the boundary in-

\footnotetext{
*Now at: Systemlab Corporation, Tokyo 171-0022, Japan.

${ }^{\dagger}$ Formerly at: Faculty of Science, Ibaraki University, Mito 310-8512, Japan.

Copyright (C) The Society of Geomagnetism and Earth, Planetary and Space Sciences (SGEPSS); The Seismological Society of Japan; The Volcanological Society of Japan; The Geodetic Society of Japan; The Japanese Society for Planetary Sciences; TERRAPUB

doi:10.5047/eps.2013.06.008
}

tegral equation method (BIEM) (e.g., Benites et al., 1992; Murai et al., 1995; Pointer et al., 1998; Kelner et al., 1999; Liu and Zhang, 2001; Yomogida and Benites, 2002). The BIEM has often been believed to have advantages over domain-type methods in computational accuracy when dealing with discrete inhomogeneities (e.g., Benites et al., 1992; Liu and Zhang, 2001), and in a great flexibility regarding the shapes of scatterers. However, it also has shortcomings: the difficulty in its application to the discrete scatterers embedded in matrices which themselves have a complex heterogeneity, computational costs that rapidly increase with the increasing numbers of scatterers, and the intractability for beginners to code their computer programs. Domain-type methods, such as the finite difference method (FDM) and the finite element method (FEM), are superior in these points.

A great advantage of the FDM is its high simplicity and tractability in general. Especially, the staggered-grid scheme (Virieux, 1984, 1986) has been known for its accuracy for media having large values of Poisson's ratio (Moczo et al., 2002). In fact, with a proper assignment of medium parameters (Ohminato and Chouet, 1997; Okamoto and Takenaka, 2005; Takenaka et al., 2009), it is now possible to simulate wave propagation in media with large material contrasts such as irregular land topography (i.e., irregular free surface), irregular ocean-bottom topography (i.e., irregular liquid-solid interface), and threedimensional heterogeneity including low-velocity soft sediments by using the staggered-grid scheme (e.g., Nakamura 
et al., 2012; Okamoto et al., 2012, 2013).

However, the incorporation of cracks into the grids is not so straightforward. Some authors modeled cracks in indirect manners, such as thin layers of equivalent homogeneous anisotropic materials (Vlastos et al., 2003) or arrayed point sources (van Antwerpen et al., 2002). In contrast, Saenger et al. (2000) modeled cracks as a thin inclusion with zero elastic constants and small mass density within the "rotated staggered grid" developed by them. An advantage of this method is the ability to treat cracks with arbitrary shapes and orientations and even with intersections. Using this method, Saenger and his coworkers have numerically investigated the effective elastic moduli of cracked media (Saenger and Shapiro, 2002; Orlowsky et al., 2003; Saenger et al., 2004, 2006). Krüger et al. (2005) also applied the method to the scattering and diffraction of an $\mathrm{SH}$ wave by a 2-D crack and demonstrated its high accuracy in comparison with an analytical solution.

The FEM has also been used for evaluating the effective elastic moduli of cracked media, though mostly adopted in simulations of elastostatic deformation rather than wave propagation (Dahm and Becker, 1998; Grechka and Kachanov, 2006; Grechka, 2007). Frehner and Schmalholz (2010) successfully applied the FEM to simulations of Stoneley guided waves around a fluid-filled fracture. Frehner et al. (2008) compared the FEM and the FDM applied to the same problem of $P-S V$ wave scattering due to a 2-D circular inclusion. They concluded that the FEM is superior to the FDM in terms of computational time because the former provides the same numerical accuracy with much less numerical grid points for modeling the curved interface of an inhomogeneity. A disadvantage of the FEM is that it is somewhat more complex to implement than the FDM and often needs a third-party software for mesh generation.

In our previous paper (Suzuki et al., 2006; hereafter referred to as Paper I), we numerically simulated $S H$ wave scattering due to traction-free, infinitesimally thin cracks by the 2-D FDM. Therein was proposed a new, simple and cost-effective simulation technique; we adopted a standard velocity-stress FDM (Virieux, 1984; Levander, 1988) and expressed cracks in a staggered grid just by arraying grid points with zero shear traction. This is a natural extension of the FDM-based simulations of crack propagation originated by Madariaga (1976), in which the boundary conditions on the crack planes can be directly given. It was much simpler than the FDM previously proposed to deal with cracked media, though its application was limited to cracks parallel to the grid lines. We proved that this technique successfully deals with $S H$ wave scattering due to cracks with practically sufficient accuracy, through accuracy tests based on the comparison with reliable BIEM estimates (Murai et al., 1995). It remained, however, to be extended to 2-D P-SV or 3-D elastic wave scattering, which would be practically more important.

An interesting topic on cracked media is the attenuation and velocity dispersion of waves propagating therein. Theoretically, they can be predicted by a stochastic theory based on Foldy's (1945) approximation. This approximation is based on the assumption of many scatterers distributed ran- domly and sparsely (Ishimaru, 1978), and it is expected to give results accurate to the first order in the distribution density (Keller, 1964). Although considerable efforts have been made to propose alternative approximations that are claimed to be valid for more densely distributed scatterers, the original Foldy approximation theory (hereafter, FAT) has maintained its popularity probably because of its mathematical simplicity (Kawahara, 2011) and the lack of consensus on which approximation is best as an alternative to the FAT (Kawahara et al., 2009). The FAT was applied to cracked media first by Kikuchi (1981a,b) and then by many authors in seismology and acoustics (Yamashita, 1990; Kawahara and Yamashita, 1992; Kawahara, 1992; Zhang and Gross, 1997; Caleap and Aristégui, 2010). However, its actual validity limit with respect to crack density remains unclear. Using their BIEM, Murai et al. (1995) simulated $S H$ wave scattering by randomly distributed 2-D cracks and determined the scattering attenuation and dispersion experimentally, thus validating the FAT of Kawahara and Yamashita (1992) as long as the crack density is relatively low $(\leq 0.02)$. Later, Murai (2007) revisited this problem and concluded that the FAT seems to be valid for a crack density up to at least 0.1 . Unfortunately, the ordinary peak picking technique, as they used in their measurement, will not work for dense crack distributions, because the initial motions of propagating waves will often be distorted too much to pick the peaks properly. In Paper I, we also performed numerical experiments like Murai et al. (1995) and Murai (2007), though we adopted a waveform averaging method in measuring the attenuation and dispersion. We showed that these parameters are obtained stably even for rather dense crack distributions, and revealed that the FAT works well for a crack density up to about 0.1 , but probably not beyond it. To the authors' knowledge, however, it has not been confirmed whether the same conclusion holds for $P-S V$ wave scattering due to 2-D cracks.

The present article is a continuation of Paper I, and its purpose is two-fold. First, we extend the FDM of Paper I in order to simulate $P-S V$ wave scattering due to 2-D tractionfree, infinitesimally thin cracks (Section 2 ). We validate the method through an accuracy test, in which it is compared with a reliable BIEM in the calculation of the displacement discontinuity along a single crack (Section 3). Second, we examine the validity of the FAT concerning $P-S V$ wave scattering due to many cracks on the basis of numerical experiments with the present simulation technique (Section 4). Discussion and conclusions are given in Section 5. As in Paper I, we treat only cracks parallel to the grid lines; the extension of the present method to the case of arbitrarily oriented cracks is discussed in Section 5.

\section{Finite Difference Method}

We adopt a 2-D velocity-stress finite difference scheme for $P-S V$ waves with the accuracy of second order in space as well as in time (Virieux, 1986). The algorithm is so standard that it is described below only briefly. The staggered grid used in the present study is shown in Fig. 1. Here, the $x$ - and $z$-axes are taken in the horizontal and vertical directions, respectively. We define $v_{x}$ and $v_{z}$ as the horizontal and vertical components of particle velocity, $\tau_{x x}$ and 


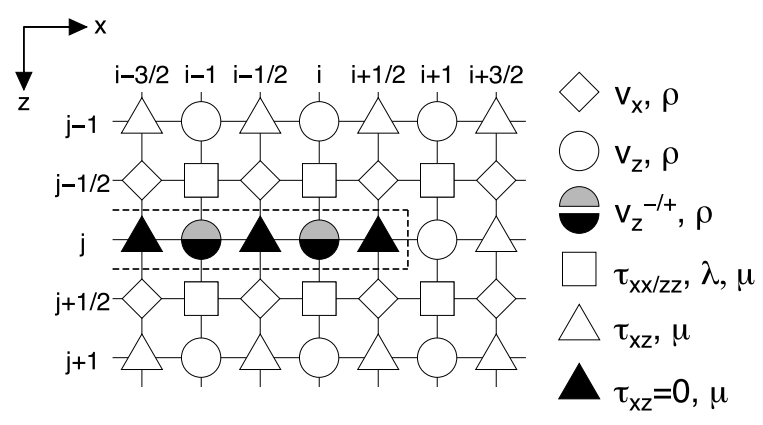

Fig. 1. Staggered grid and implementation of cracks. Different symbols denote grid points on which different quantities are allocated as follows; diamonds: horizontal particle velocity and mass density; circles and semicircles: vertical particle velocity and mass density; squares: normal stress, $\lambda$, and $\mu$; triangles: shear stress and $\mu$. Here, $\lambda$ and $\mu$ are the Lamé parameters. The region enclosed by the dotted line corresponds to a crack, in which the solid triangles denote the points with zero shear stress. Each pair of gray and black semicircles denotes two close points located on the upper and lower crack faces, respectively, whose separation is much smaller than the grid spacing.

$\tau_{z z}$ as normal stress, $\tau_{x z}$ as inplane shear stress, $\lambda$ and $\mu$ as the Lamé parameters, and $\rho$ as mass density. The grid spacings in the $x$ - and $z$-directions are assumed to have the same length $\Delta h$. Letting the time step be $\Delta t$, the finite difference scheme for this grid is expressed as:

$$
\begin{gathered}
v_{x}\left(i-\frac{1}{2}, j-\frac{1}{2}, m+\frac{1}{2}\right)=v_{x}\left(i-\frac{1}{2}, j-\frac{1}{2}, m-\frac{1}{2}\right) \\
+\frac{1}{\rho\left(i-\frac{1}{2}, j-\frac{1}{2}\right)} \frac{\Delta t}{\Delta h} \\
\times\left[\tau_{x x}\left(i, j-\frac{1}{2}, m\right)-\tau_{x x}\left(i-1, j-\frac{1}{2}, m\right)\right. \\
\left.+\tau_{x z}\left(i-\frac{1}{2}, j, m\right)-\tau_{x z}\left(i-\frac{1}{2}, j-1, m\right)\right], \\
v_{z}\left(i, j, m+\frac{1}{2}\right)=v_{z}\left(i, j, m-\frac{1}{2}\right)+\frac{1}{\rho(i, j)} \frac{\Delta t}{\Delta h} \\
\times\left[\tau_{z z}\left(i, j+\frac{1}{2}, m\right)-\tau_{z z}\left(i, j-\frac{1}{2}, m\right)\right. \\
\left.\quad+\tau_{x z}\left(i+\frac{1}{2}, j, m\right)-\tau_{x z}\left(i-\frac{1}{2}, j, m\right)\right], \\
\tau_{x x}\left(i, j-\frac{1}{2}, m+1\right)=\tau_{x x}\left(i, j-\frac{1}{2}, m\right)+\frac{\Delta t}{\Delta h} \\
\times\left[M ( i , j - \frac { 1 } { 2 } ) \left\{v_{x}\left(i+\frac{1}{2}, j-\frac{1}{2}, m+\frac{1}{2}\right)\right.\right. \\
\left.-v_{x}\left(i-\frac{1}{2}, j-\frac{1}{2}, m+\frac{1}{2}\right)\right\}+\lambda\left(i, j-\frac{1}{2}\right) \\
\left.\times\left\{v_{z}\left(i, j, m+\frac{1}{2}\right)-v_{z}\left(i, j-1, m+\frac{1}{2}\right)\right\}\right], \\
\tau_{z z}\left(i, j-\frac{1}{2}, m+1\right)=\tau_{z z}\left(i, j-\frac{1}{2}, m\right)+\frac{\Delta t}{\Delta h} \\
\times\left[\lambda ( i , j - \frac { 1 } { 2 } ) \left\{v_{x}\left(i+\frac{1}{2}, j-\frac{1}{2}, m+\frac{1}{2}\right)\right.\right. \\
\left.-v_{x}\left(i-\frac{1}{2}, j-\frac{1}{2}, m+\frac{1}{2}\right)\right\}+M\left(i, j-\frac{1}{2}\right) \\
\left.\times\left\{v_{z}\left(i, j, m+\frac{1}{2}\right)-v_{z}\left(i, j-1, m+\frac{1}{2}\right)\right\}\right],
\end{gathered}
$$

$$
\begin{gathered}
\tau_{x z}\left(i-\frac{1}{2}, j, m+1\right)=\tau_{z z}\left(i-\frac{1}{2}, j, m\right)+\frac{\Delta t}{\Delta h} \mu\left(i-\frac{1}{2}, j\right) \\
\times\left[v_{x}\left(i-\frac{1}{2}, j+\frac{1}{2}, m+\frac{1}{2}\right)-v_{x}\left(i-\frac{1}{2}, j-\frac{1}{2}, m+\frac{1}{2}\right)\right. \\
\left.+v_{z}\left(i, j, m+\frac{1}{2}\right)-v_{z}\left(i-1, j, m+\frac{1}{2}\right)\right], \quad(1 \mathrm{e})
\end{gathered}
$$

in which $M=\lambda+2 \mu$ and $(i, j, m)$ denotes the association with the grid point $(i, j)$ and the time $t=m \Delta t$.
We assume for the moment that cracks are horizontal (parallel to the $x$-axis). We impose the traction-free boundary condition $\tau_{x z}=\tau_{z z}=0$ on the crack planes. The cracks are incorporated into the grid in the following manner. First, we impose $\tau_{x z}=0$ on a sequence of horizontally arrayed grid points (solid triangles in Fig. 1) which define a crack plane. The crack tips are defined as the leftmost and rightmost points of the array (e.g., the grid point $\left(i+\frac{1}{2}, j\right)$ in the figure). However, we cannot explicitly impose $\tau_{z z}=0$ on the crack plane thus defined, because the crack is composed of grid points for $\tau_{x z}$ and for $v_{z}$ but not for $\tau_{z z}$. Such a difficulty was not encountered in Paper I, in which it was enough to let antiplane shear stress $\tau_{x y}$ only be zero. Alternatively, we split here each grid point for $v_{z}$ on the crack into two very close points, which respectively represent the points on the upper and lower crack faces (the gray and black semicircles in Fig. 1). Since there arises a displacement discontinuity (Section 3), incorporation of such a split variable, or a "two-valued" variable, is inevitable in order to correctly express the response of an infinitesimally thin crack when the grid points for particle velocity (or displacement) are located on the crack plane. We express the coordinates of these points as, say, $(i, j \pm \delta)$, in which $\delta$ may be regarded as half of the infinitesimal crack width with $0<\delta \ll \Delta h$. Introducing further the notation as $v_{z}\left(i, j \pm \delta, m-\frac{1}{2}\right)=v_{z}^{ \pm}\left(i, j, m-\frac{1}{2}\right)$, we calculate $v_{z}^{ \pm}\left(i, j, m+\frac{1}{2}\right)$ by the following scheme with either a forward or backward difference with regard to $\tau_{z z}$ as:

$$
\begin{gathered}
v_{z}^{ \pm}\left(i, j, m+\frac{1}{2}\right)=v_{z}^{ \pm}\left(i, j, m-\frac{1}{2}\right) \pm \frac{1}{\rho(i, j)} \frac{\Delta t}{3 \Delta h} \\
\times\left[9 \tau_{z z}\left(i, j \pm \frac{1}{2}, m\right)-\tau_{z z}\left(i, j \pm \frac{3}{2}, m\right)\right] .
\end{gathered}
$$

This scheme satisfies the boundary conditions on the crack planes as described in Appendix A. Note that Eq. (1b) requires the continuity of the displacement and, hence, it is only applicable to media without cracks. A similar equation to Eq. (2) was previously derived by Hayashida et al. (1999) to express a free surface in a staggered grid for 3-D elastic media.

Then, in the vicinity of the crack, the normal stress $\tau_{x x}\left(i, j \pm \frac{1}{2}, m+1\right)$ and $\tau_{z z}\left(i, j \pm \frac{1}{2}, m+1\right)$ are calculated using $v_{z}^{ \pm}\left(i, j, m+\frac{1}{2}\right)$ instead of $v_{z}\left(i, j, m+\frac{1}{2}\right)$ in Eqs. (1c) and (1d). Note that vertical cracks (parallel to the $z$-axis) can be also treated in almost the same manner. Although the above-mentioned manner to incorporate cracks into a grid may not be so simple as that for the case of $S H$ wave scattering given in Paper I, it still seems to retain simplicity as compared with those of other previous studies (Section 1).

In any simulation performed below, a plane wavelet is assumed to propagate upward (in the negative $z$-direction) from below and be incident on cracks either normally or parallel. Then, velocity seismograms are synthesized at specified grid points, which are finally integrated with the trapezoidal rule to yield the displacement seismograms. As in Paper I, $\Delta h$ and $\Delta t$ are chosen to satisfy the stability condition $\Delta t<\Delta h / \sqrt{2} \alpha$ and the sampling criterion $\Delta h<$ $\beta / n f_{u l}$ (Virieux, 1986; Moczo, 1998), in which $n=10$ for the second-order scheme, $\alpha=\sqrt{M / \rho}$ and $\beta=\sqrt{\mu / \rho}$ are the $P$ and $S$ wave velocity of the matrix, respectively, and 
$f_{u l}$ is the frequency up to which one needs to have accurate results without the grid dispersion. For convenience, $\beta$, $\rho$, and the crack half length $a$ are set to be unity and the values of other parameters are normalized with them. We also assume a Poisson solid with $\lambda=\mu$ and, hence, $\alpha=$ $\sqrt{3} \beta$. Concerning the artificial boundaries of the whole grid (model space), we impose again simple conditions. The cyclic boundary condition is applied to the left and right ends to express approximately an infinitely long cracked layer. A standard absorbing boundary condition of Clayton and Engquist (1977) is assumed along the top and bottom ends. Although artificial reflections from the absorbing boundaries cannot be perfectly erased, we actually analyze the portions of seismograms not contaminated with them.

\section{Crack Displacement Discontinuity}

As in Paper I, we compare the results of our FDM simulations with those based on a frequency-domain BIEM, thus trying to validate the present simulation method. For this purpose, we calculate the displacement discontinuity along a single crack caused by incident harmonic waves, using both methods. Note that the normal displacement discontinuity (NDD) along a traction-free, infinitesimally thin crack due to normally incident harmonic $P$ waves was analytically solved by Mal (1970). Kawahara and Yamashita (1992) developed a BIEM algorithm for treating an isolated crack, which reproduces Mal's results quite excellently. Hence, we infer their BIEM to be reliable and adopt it here. In order to treat the BIEM results as the correct answers, we make the discretization interval of the crack planes in the BIEM calculation to be much smaller than the crack length as well as the wavelength, as in Paper I.

The manner of the FDM calculation of the crack displacement discontinuity is the same as that in Paper I. Consider an isolated horizontal crack with the length $2 a$. Let a quasi-monochromatic wave train

$$
u^{0}(x, z, t)=b H(l z+\omega t) \sin (l z+\omega t)
$$

propagate upward and then impinge normally on the crack, in which $b$ is the amplitude, $\omega$ is the angular frequency and $H()$ is the Heaviside step function. The wave mode is either $P$ or $S V$; $l$ and $u^{0}$, respectively, denote the $P$ wavenumber $h=\omega / \alpha$ and the vertical displacement for the former, whereas they denote the $S$ wavenumber $k=\omega / \beta$ and the horizontal displacement for the latter. Note that $h=k / \sqrt{3}$ because $\alpha=\sqrt{3} \beta$. The wave incidence causes the oscillation of the crack faces, which approaches the stationary state with the increasing lapse time. We evaluate the amplitudes of the oscillatory displacement discontinuity along the crack when the oscillation appears almost stationary. Since the shear displacement discontinuity (SDD) cannot be defined directly at the grid points on the crack plane, we instead measure the differences of the $x$-component displacement seismograms calculated at the pairs of points just above and below the plane (e.g., the points $\left(i-\frac{1}{2}, j \pm \frac{1}{2}\right)$ in Fig. 1), and then take their amplitudes. The approximation errors due to the offsets of the measurement points from the crack plane would be small if $\Delta h$ is sufficiently small. In contrast, the NDD can be defined directly on the crack plane (e.g., the grid point $(i, j)$ in Fig. 1); that is, it is calculated

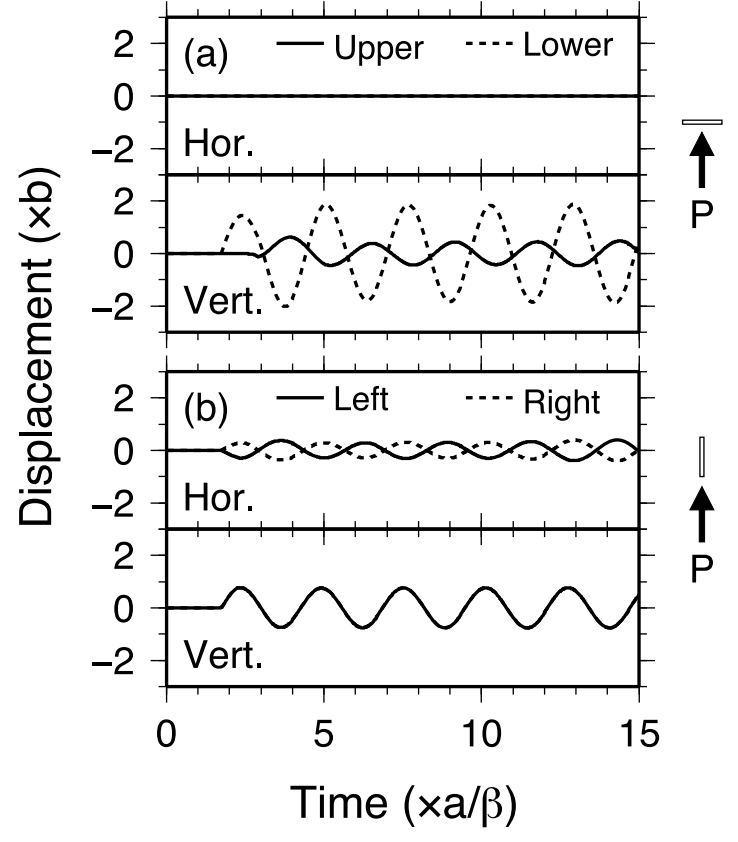

Fig. 2. Oscillation of both faces of an isolated crack due to the incidence of a quasi-monochromatic $P$ wave train $(k a=2.4, h a=1.39)$ observed at around the center of the crack. The panels with 'Hor.' and 'Vert.' indicate horizontal and vertical displacement, respectively. (a) Seismograms for a horizontal crack due to normal wave incidence. Solid and dashed curves correspond to the upper (shaded) and lower (illuminated) faces, respectively. In the upper panel, the solid curve masks the dashed one. (b) Seismograms for a vertical crack due to parallel wave incidence. Solid and dashed curves correspond to the left and right crack faces, respectively. In the lower panel, the solid curve masks the dashed one.

from the time integration of $v_{z}^{+}-v_{z}^{-}$at the same points. We also examine the case of an isolated vertical crack, on which waves impinge parallel to the crack. The NDD and SDD along the crack are then defined in a similar manner.

We set here $\Delta t=0.003 a / \beta$ and $\Delta h=0.0125 a$. Choosing the same values of the parameters, the second-order FDM adopted in Paper I gave an SDD which was highly consistent with those by the BIEM, independently of the wavenumbers. Note that the fourth-order FDM was shown to systematically underestimate the SDD (by several percent), probably because of the nonlocality of the finite difference operator; see Paper I as to the detailed discussion. We use here the second-order FDM throughout. Note also that the present choice of $\Delta h$ and the sampling criterion (Section 2) require an inequality $f_{u l}<8.0 \beta / a$ whereas the incident waves considered here (and also in Section 4) are shown to have a negligibly small energy for a frequency beyond $8.0 \beta / a$ (corresponding to $k a>50$ ). This implies that every simulation will not suffer grid dispersion.

Figure 2 gives examples of the simulated oscillation of both the faces of an isolated crack, on which a quasi-monochromatic $P$ wave train with the normalized $S$ wavenumber $k a=2.4$ (normalized $P$ wavenumber $h a=$ 1.39) is incident. They are the horizontal- and verticalcomponent seismograms recorded at grid points adjacent to the center of the crack. We see that the oscillation begins and then becomes almost stationary within a few periods 


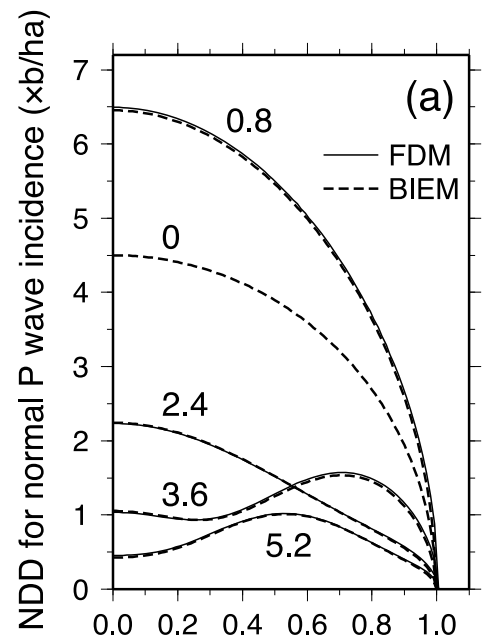

Distance along the crack $(\times a)$

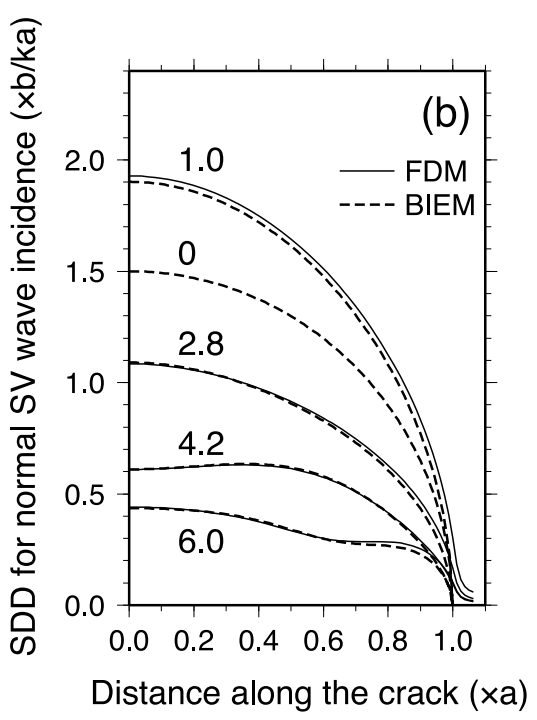

Fig. 3. Displacement discontinuity along an isolated crack due to a normal incidence of harmonic $P$ or $S V$ waves: (a) normal displacement discontinuity due to $P$ waves, and (b) shear displacement discontinuity due to $S V$ waves. The abscissas represent the distance from the center of the crack. The curve parameters denote $\mathrm{ka}$. The solid and dashed lines indicate the calculations based on the present FDM and the BIEM of Kawahara and Yamashita (1992), respectively.

of the incident wave. The NDD or SDD will be obtained from the difference of the pair of seismograms in each panel. We notice that the normal incidence of the $P$ wave on the horizontal crack causes only a vertical displacement on the crack faces (Fig. 2(a)). In contrast, the parallel incidence of the $P$ wave on the vertical crack makes the crack faces oscillate vertically as well as horizontally, though the vertical displacement of both crack faces are always equal (Fig. 2(b)). These features are recognized irrespective of the positions along the crack and the wavenumbers of the incident waves, implying that the SDD never occurs for $P$ waves incident either normal or parallel to a crack, as expected. Similarly, we also confirmed that the NDD never occurs for either a normal or parallel incidence of $S V$ waves (figure omitted).

In the following, we show only the results on non-zero NDD and SDD. Their values are normalized with $h$ and $k$ for $P$ and $S$ wave incidence, respectively, after Mal (1970). Note that the same normalization was also employed in figure 2 of Paper I, but without mention.

Figure 3(a) denotes the NDD due to the normal $P$ wave incidence for the normalized $S$ wavenumber $k a=0,0.8$, 2.4, 3.6, and 5.2 (normalized $P$ wavemunber $h a=0,0.46$, $1.39,2.08$, and 3.00, respectively) after Mal (1970); the corresponding $P$ wavelengths are $\infty, 13.6 a, 4.5 a, 3.0 a$, and $2.1 a$, respectively. Since the displacement discontinuity for $k a=0$ cannot be calculated by the present time-domain FDM, we show only the BIEM estimates in this static case. Note that the discontinuity is always symmetrical with respect to the center of the crack for normal wave incidence. The figure shows good agreement between the FDM and BIEM estimates for every positive $k a$.

Figure 3(b) denotes the SDD due to the normal $S V$ wave incidence for the normalized $S$ wavenumber $k a=0,1.0$, 2.8, 4.2, and 6.0; the corresponding $S$ wavelengths are $\infty$, $6.3 a, 2.2 a, 1.5 a$, and $1.0 a$, respectively. Note that the same values of $k a$ were chosen in Paper I for the accuracy test of its FDM calculations after Mal (1970). In this case, the discontinuity by the FDM does not vanish outside the crack (at a distance larger than 1.0 $a$ in the figure). As mentioned in Paper I, this can be explained by the offsets of the measurement points from the crack plane, and, therefore, gets smaller for smaller $\Delta h$. Thus, it does not imply the failure of the present FDM. Except for this, the agreement between the FDM and BIEM estimates is satisfactory also in this case. Note that the agreement seems slightly worse than that seen in Fig. 3(a), especially for $k a=1$. This may also be explained by the offsets of the measurement points stated above.

The results on NDD and SDD for parallel $P$ and $S V$ wave incidence are shown in Figs. 4(a) and 4(b), respectively. In these cases, the discontinuity is not symmetrical with respect to the center of the crack except for the static limit $(k a=0)$, so that it needs to be displayed over the whole crack. The overall features of the figures are the same as those seen in Figs. 3(a) and 3(b); the FDM and BIEM estimates are again consistent for both $P$ and $S V$ wave incidence and for every positive wavenumber.

In summary, the present FDM produces the crack displacement discontinuity due to either $P$ or $S V$ wave incidence fairly accurately, independently of wavenumbers. We therefore conclude that the present method is applicable to the synthesis of $P-S V$ waves scattered by cracks.

\section{Validation of the Foldy Approximation Theory}

In this section, we show an example of the application of the present FDM, whose accuracy has been confirmed above. Using the method, we investigate here the validity of the FAT of Kawahara (1992), which predicts the scattering attenuation and velocity dispersion of $P-S V$ waves in 2-D media with identical parallel cracks. Here, the prediction is stochastic in the sense that the theory is based on the mean wave formalism; i.e., it treats the ensemble average of real wavefields. Note that the theory also adopts an ap- 

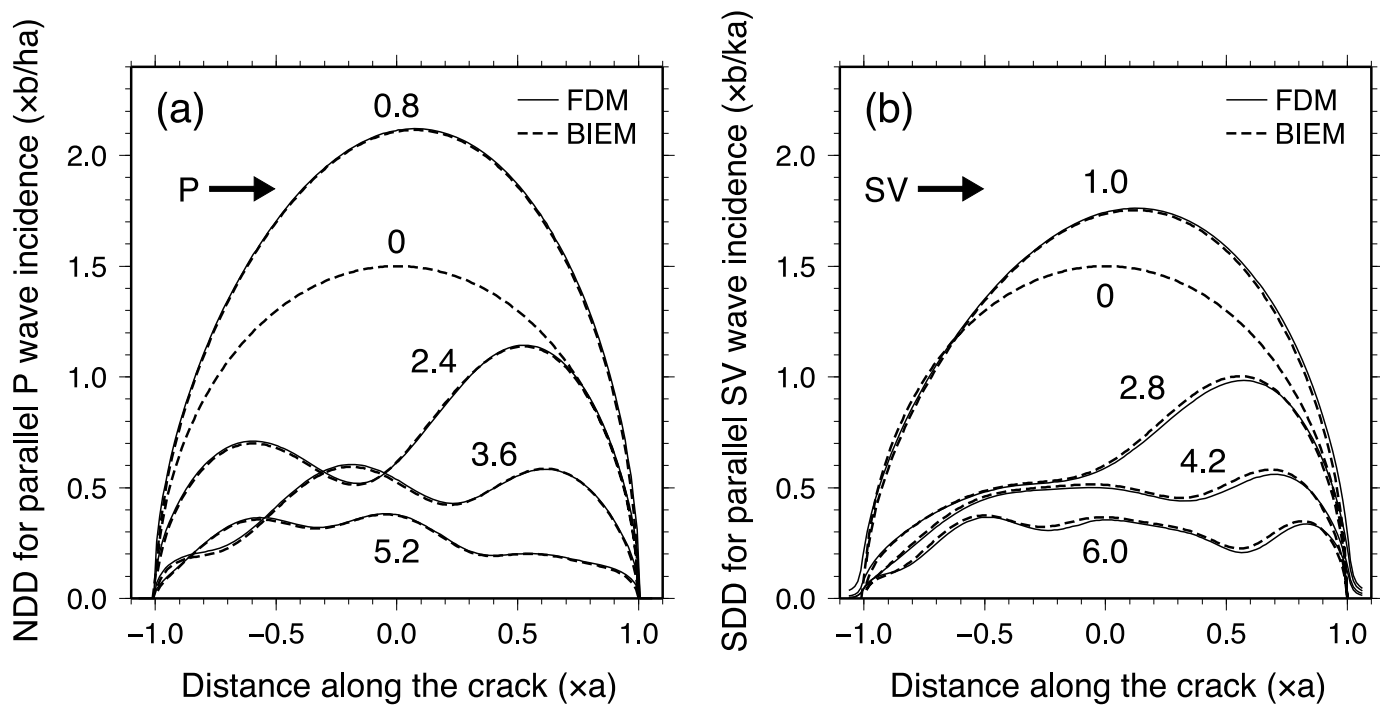

Fig. 4. Displacement discontinuity along an isolated crack due to a parallel incidence of harmonic $P$ or $S V$ waves: (a) normal displacement discontinuity due to $P$ waves, and (b) shear displacement discontinuity due to $S V$ waves. The abscissas represent the positions relative to the center of the crack. The rightward arrows indicate the direction of wave propagation relative to the crack plane. The curve parameters denote $k a$. The solid and dashed lines indicate the calculations based on the present FDM and the BIEM of Kawahara and Yamashita (1992), respectively.

Table 1. Parameters of the crack distribution models used in the simulations. $P$ and $S V$ indicate the wave modes. $H$ and $V$ indicate the horizontal and vertical cracks (normal and parallel wave incidence), respectively.

\begin{tabular}{cccccr}
\hline Model & Wave/Cracks & $N$ & $W \times L$ & \multicolumn{1}{c}{$\epsilon$} & $N_{D}$ \\
\hline 1 & $P / H, P / V, S V / H, S V / V$ & 19 & $40 a \times 38 a$ & 0.0125 & 6 \\
2a & $P / H, S V / H, S V / V$ & 36 & $40 a \times 18 a$ & 0.05 & 12 \\
$2 \mathrm{~b}$ & $P / V$ & 76 & $40 a \times 38 a$ & 0.05 & 12 \\
3a & $P / H, S V / H, S V / V$ & 32 & $40 a \times 8 a$ & 0.1 & 18 \\
3b & $P / V$ & 152 & $40 a \times 38 a$ & 0.1 & 18 \\
\hline
\end{tabular}

proximation in which the waves effectively impinging on each scatterer (crack) are equal to the waves that would be observed without the scatterer. This approximation, first introduced by Foldy (1945), yields a closed equation for mean waves, called the Foldy-Twersky integral equation (Ishimaru, 1978). Kawahara and Yamashita (1992) solved it for $P-S V$ as well as $S H$ wave scattering due to cracks filled with an incompressible viscous fluid, and obtained the attenuation and phase velocity of the mean waves to the first order in the crack density. Kawahara (1992) then analyzed the case for $P-S V$ wave scattering due to empty (traction-free) cracks. In Paper I, we validated in a numerical-experimental manner the FAT of Kawahara and Yamashita on $\mathrm{SH}$ wave scattering in the case of cracks filled with an inviscid fluid (or equivalently, shear stress-free cracks); the same experimental procedures are again adopted here, which are summarized below.

\subsection{Outline of the simulations}

First, we assume $N$ cracks with a length of $2 a$ inside a rectangular area with a horizontal size of $W$ and vertical size of $L$. The cracks are distributed in such a manner as visualized in figure 8 of Paper I; that is, they are located almost randomly, except for the non-overlapping constraint that the interval between any two points belonging respectively to any two crack planes must be greater than $0.15 a$. Here, the 2-D crack density is defined as $\epsilon=N a^{2} / W L$. Second, we array 100 receivers evenly along a horizontal line $2 a$ above the top end of the crack distribution area. We then let a plane wavelet be vertically incident on the bottom end from below, and synthesize seismograms at the receivers using the FDM. We adopt here an incident wavelet whose source time function is given as the first derivative of a Gaussian function; see equations (4), (5) and figure 7 of Paper I as to the details. The peak frequency of its power spectrum is $1 / \sqrt{\pi} \approx 0.56$ in the unit of $\beta / a$, corresponding to $k a=2 \sqrt{\pi} \approx 3.5$ and $h a=2 \sqrt{\pi / 3} \approx 2.0$. Third, we average the seismograms for all the receivers to obtain a "spatial-averaged seismogram". Here, we assume ergodicity, i.e., we assume that ensemble averaging can be replaced with spatial averaging (Shapiro and Kneib, 1993). Fourth, we repeat the simulation and averaging process as stated above for $N_{D}$ different realizations of the crack distribution that are stochastically identical but are generated using different random number series. Thus, $N_{D}$ spatialaveraged seismograms are obtained from these realizations of the crack distribution. We then take an average of these spatial-averaged seismograms; the finally obtained result is termed the "synthetic mean wave". Fifth, the spectrum of the synthetic mean wave is calculated. In doing this, we select a time window that effectively includes the whole synthetic mean wave. If the averaging process is so successful that the incoherent coda waves have been removed almost completely, the window width will not influence the results. We then evaluate the attenuation $Q^{-1}$ and the phase velocity 
$v$ from the spectral ratio of the synthetic mean wave to the initial wavelet that would be observed without the cracks, $C(\omega)$; after some calculation, we obtain:

$$
C(\omega)=\exp \left[i \omega L\left(\frac{1}{v}-\frac{1}{\beta}\right)-\frac{\omega L}{2 v Q}\right],
$$

and, hence,

$$
\begin{gathered}
\frac{1}{v}=\frac{1}{\beta}+\frac{1}{\omega L} \tan ^{-1} \frac{\operatorname{Im} C}{\operatorname{Re} C}, \\
Q^{-1}=-\frac{2 v}{\omega L} \log |C|,
\end{gathered}
$$

in which "Im" and "Re" denote the imaginary and real parts, respectively. The obtained $Q^{-1}$ and $v$ are to be compared with the values predicted by the FAT under the assumption that the mean wave is of the same mode (either $P$ or $S V$ ) as that of the initial wavelet. Practically, we evaluate these quantities only for some frequency band inside which the initial wavelet has a sufficiently high energy for the sake of an accurate calculation. Instead of evaluating the estimation errors of $Q^{-1}$ and $v$ directly, we calculate the standard deviation of the synthetic mean wave and regard it as the error range. We also compute the waveform predicted by the FAT ("predicted mean wave"). If the predicted mean wave coincides with the synthetic mean wave within the error range, we regard their coincidence as significant.

In every simulation performed, we set $\Delta t=0.003 a / \beta$ and $\Delta h=0.0125 a$ as in the preceding section. Other parameters of the crack distribution models used are summarized in Table 1. An example of the snapshot of a simulated wavefield is shown in Fig. 5, in which scattered waves from individual cracks are clearly seen.

We notice that Shapiro and Kneib (1993) have stated that spatial averaging works well if the length of the receiver array is much larger than $\max \left(a_{c l}, \lambda_{w l}\right)$, in which $a_{c l}$ is the correlation length of the medium heterogeneity and $\lambda_{w l}$ is a wavelength; in such cases, the incoherent part of a wavefield would interfere destructively during the spatial averaging process and only the coherent part (mean wave) would be retained. In the present simulation geometry, $a_{c l}$ nearly corresponds to the size of the scatterers (cracks) $2 a$ (Kawahara, 2011), which is much smaller than the length of the receiver array $W=40 a$ (Table 1). However, the wavelengths are not always sufficiently smaller than $W$. As seen in the following subsections, we will numerically evaluate $Q^{-1}$ and $v$ for $0.5<k a<10(0.29<h a<5.77)$ in which the initial wavelet has a high energy. This wavenumber range corresponds to $21.8 a>\lambda_{P}>1.1 a$ and $12.6 a>\lambda_{S}>0.6 a$, in which $\lambda_{P}$ and $\lambda_{S}$ are $P$ and $S$ wavelengths, respectively. Hence, $W$ is only a few times larger than the maximum wavelength of either the $P$ or the $S$ wave corresponding to $k a=0.5$. As a result, the spatial averaging of seismograms for only one realization of a crack distribution removes the incoherent waves only partially, and, hence, the ensemble averaging process among many realizations is required for the total removal of the incoherent waves. Actually, we chose $N_{D}$ in Table 1 to achieve this purpose by increasing $N_{D}$ until coda-like wave trains effectively vanish from the synthetic mean wave. (a) Vertical

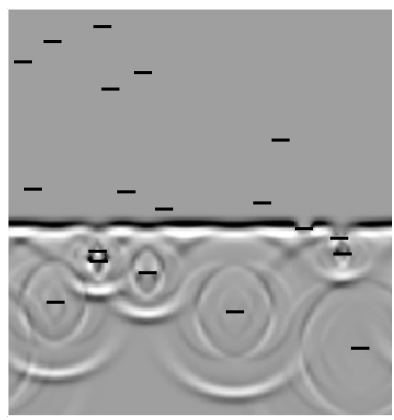

(b) Horizontal

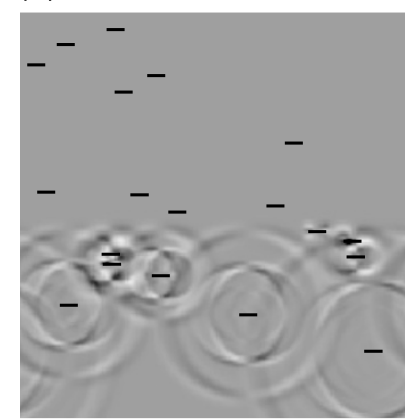

Fig. 5. Snapshot of a $P$ wave propagating upward through a cracked region (a realization of Model 1). Black and white indicate positive and negative values of displacement, respectively. Short horizontal black lines indicate cracks. (a) Vertical and (b) horizontal displacement.

\subsection{Results for sparsely distributed cracks}

We first demonstrate the results for Model 1, with which the crack density is so low $(\epsilon=0.0125)$ that the FAT is expected to be sufficiently valid. The result for the case of $P$ wave incidence on horizontal cracks is shown in Fig. 6 . In Figs. 6(a) and (b), we plot $N_{D} \times 100$ horizontal- and vertical-component seismograms, respectively. Here, we also show the traces of the synthetic mean wave and its standard deviation (error range) derived from the seismograms; the initial wavelet without the cracks is also plotted for reference. In Fig. 6(a) (see also Fig. 6(c) as the close-up), one may clearly observe the attenuation on the primary part of the synthetic mean wave $(25<t<27$ in unit of $a / \beta)$. The primary wave is also followed by a pulse of relatively long duration (around $27<t<32$ ). However, the trace does not contain a coda-like long wave train, implying that the averaging process has successfully removed coda waves. We further plot the trace of the predicted mean wave in the figure. The agreement between the synthetic and predicted mean waves is considerably good. This also suggests that the above-mentioned pulse after the primary wave would be attributed to the waveform dispersion of the initial wavelet due to scattering. Note that such a feature of the wave traces was previously reported in Paper I for $S H$ wave incidence (figure 9 thereof). In contrast, Fig. 6(b) indicates that the horizontal component of the synthetic mean wave continues to be effectively zero despite the high amplitude of each synthetic seismogram. This validates the assumption of the FAT that the modes of the mean wave and the initial wavelet are the same (Subsection 4.1), though $P$-to- $S$ conversion scattering significantly occurs.

The values of $Q^{-1}$ and $\Delta v$ derived from the synthetic and predicted mean waves in Fig. 6(a) are plotted in Figs. 6(d) and $6(\mathrm{e})$ for $0.5<k a<10$, respectively, in which $\Delta v$ is the phase velocity reduction due to scattering; it is defined as either $\Delta v=\alpha-v$ or $\Delta v=\beta-v$, depending on the modes of the incident waves. The agreement between the experimental values (from the synthetic mean wave) and the theoretical values (from the predicted mean wave) are fairly good on the whole, even including the ripple pattern of $\Delta v$ for high wavenumbers, as is expected from the good agreement between the synthetic and predicted mean waves 

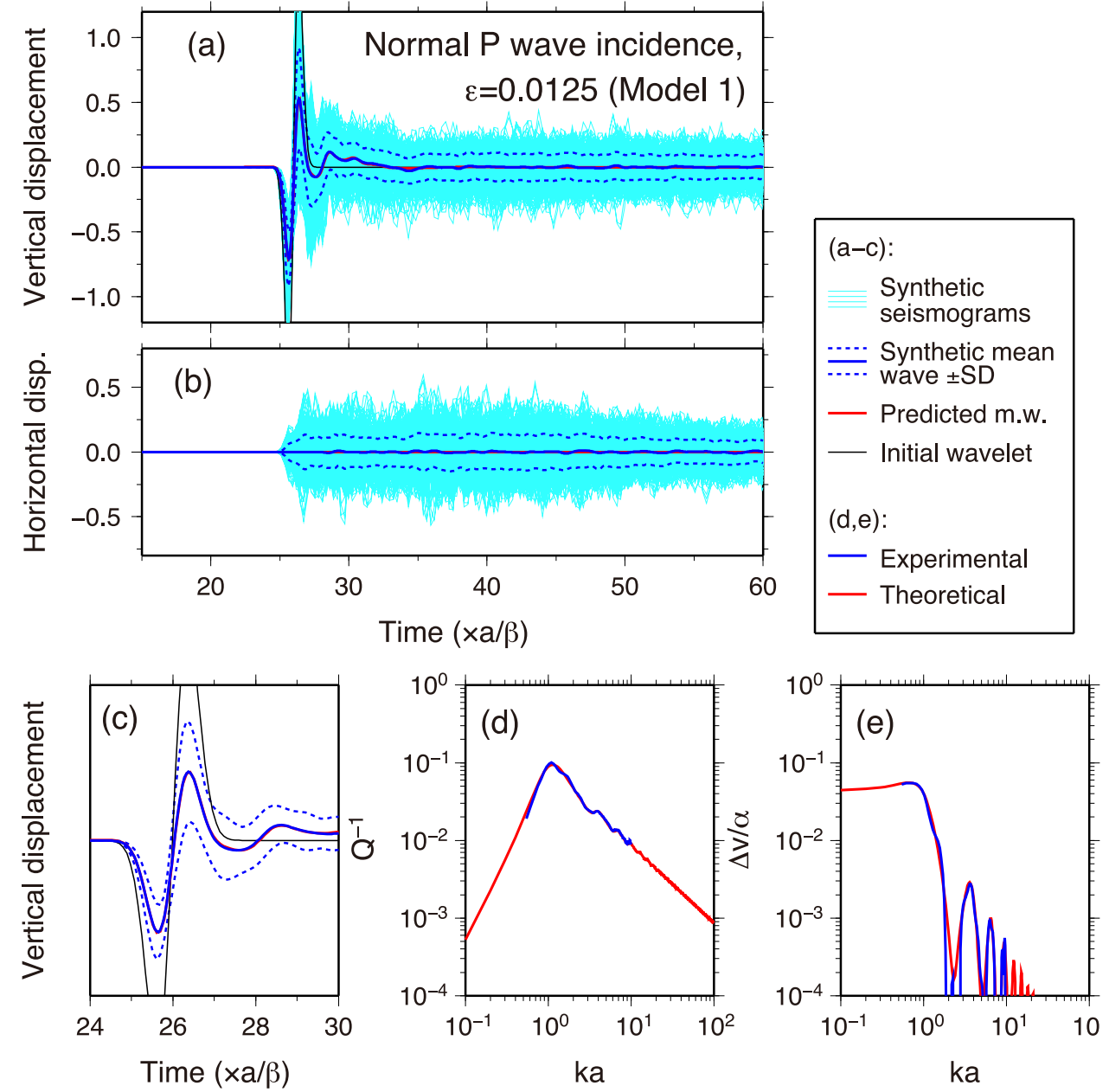

Fig. 6. (Color online) Results of the simulations obtained for Model $1(\epsilon=0.0125)$ with $P$ wave incidence on horizontal cracks. (a) Vertical- and (b) horizontal-component synthetic seismograms (the cloud of thin light blue lines), and the synthetic mean wave and its standard deviation derived from these seismograms (solid and dotted blue curves, respectively). The predicted mean wave is indicated by the red curve, though almost masked by the solid blue one. The thin black curve denotes the initial wavelet that would be observed without the cracks. (c) The close-up of the primary part in (a). The synthetic seismograms are omitted. (d) $Q^{-1}$, and (e) $\Delta v$ for the synthetic mean wave (blue curves). The prediction by the FAT is denoted by red curves.

in Fig. 6(a).

The result for the same model but for the case of $S V$ wave incidence on horizontal cracks is depicted in Fig. 7. A noticeable feature of Fig. 7(a) is that incoherent wave motion exists at each synthetic seismogram prior to the arrival of the primary $S V$ wave. This motion will be attributed to $S$ to- $P$ conversion scattering due to cracks; after averaging, it effectively vanishes from the trace of the synthetic mean wave, just as the later coda waves. Except for this difference, the overall features of Fig. 7 are quite similar to those of Fig. 6: the mean wave is of the same mode as that of the initial wavelet, and its waveform, attenuation, and dispersion are described by the FAT satisfactorily.

Figure 8 shows the results for the same model but for the case of $P$ - and $S V$-waves incident on vertical cracks, supporting again the validity of the FAT for such sparse distributions of cracks. It might be interesting that the dispersive deformation of the mean waveforms is rather significant for $S V$ wave incidence on vertical cracks (Fig. 8(d)). In this case, a new peak, which is not recognized for the initial wavelet, emerges prior to the primary trough (at around $t=44.2$ ). This may be related to the somewhat abnormal shapes of the curves of $Q^{-1}$ and $\Delta v$ indicated in Figs. 8(e) and $8(\mathrm{f})$.

\subsection{Results for more densely distributed cracks}

We next show the results for the models with denser distributions (Models 2 and 3), focusing on the validity limit of the FAT.

Figure 9 depicts the results for Model 2 ( $2 \mathrm{a}$ or $2 \mathrm{~b}$ ) with a crack density $\epsilon=0.05$. Note that the case of $P$ wave incidence on vertical cracks was examined on the basis of Model $2 \mathrm{~b}$ unlike the other three cases, which has more cracks and a wider space than Model $2 \mathrm{a}$ adopted for the latter cases (Table 1). It is because that the $Q^{-1}$ and $\Delta v$ are rather small in this case, as indicated by Figs. 9(h) and 9(i), so that a large travel distance seems to be preferable for measuring them. In Fig. 9, we may still recognize the fairly good agreement between the experimental and theoretical results on the whole. An exception is the case of $P$ wave incidence on the vertical cracks, in which a notable discrepancy exists between the experimental and theoretical values of either $Q^{-1}$ or $\Delta v$ (Figs. 9(h) and 9(i)). Nevertheless, the corresponding traces of the synthetic and predicted mean waves continue to agree fairly well (Fig. 9(g)). This phe- 

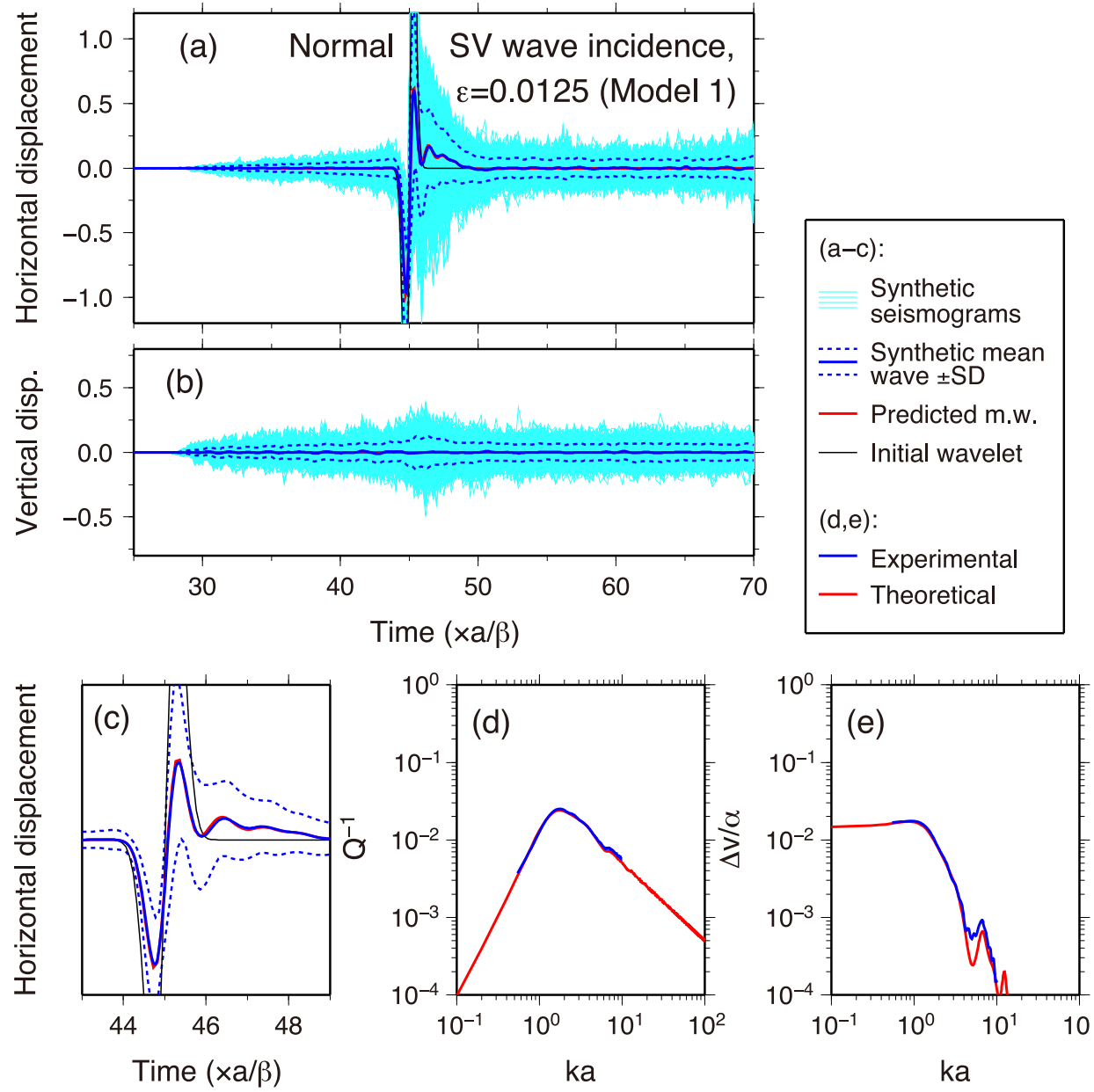

Fig. 7. (Color online) Results of the simulations obtained for Model $1(\epsilon=0.0125)$ with an $S V$ wave incidence on horizontal cracks, shown in the same manner as in Fig. 6. (a) Horizontal- and (b) vertical-component synthetic seismograms, and the synthetic mean wave and its standard deviation derived from these seismograms. The synthetic mean wave and initial wavelet, if without the cracks, are also depicted. (b) The close-up of the primary part in (c), though without the synthetic seismograms. (d) $Q^{-1}$, and (e) $\Delta v$ for the synthetic and predicted mean waves.

nomenon may be explained by the fact that the discrepancy between the experimental and theoretical values of $Q^{-1}$ or $\Delta v$ is mainly limited to $k a<2$, whereas both the synthetic and predicted mean waves can be shown to have a high energy for $1.5<k a<4$. This implies that the waveforms of both mean waves are dominated by their respective spectral contents in the range $1.5<k a<4$. Furthermore, these two spectral contents are similar because the experimental and theoretical values of $Q^{-1}$ or $\Delta v$ are close in this wavenumber range. In contrast, the primary peaks and troughs of the mean waves are somewhat overestimated by the FAT for horizontal cracks (Figs. 9(a) and 9(d)), which may correspond to the slight underestimation of $Q^{-1}$ by the FAT at around $k a=3.5$ (Figs. 9(b) and 9(e)).

The results for Model 3 ( $3 \mathrm{a}$ or $3 \mathrm{~b}$ ) with a higher crack density $(\epsilon=0.1)$ are depicted in Fig. 10. Now the overestimation of $Q^{-1}$ and $\Delta v$ by the FAT for $k a<2$ is rather remarkable for $P$ wave incidence on the vertical cracks (Figs. 10(h) and 10(i)). In this case, the trace of the predicted mean wave almost deviates from the error range of the synthetic mean wave, though temporarily (at around $t=27.5$ ) (Fig. 10(g)). The same tendency is also recognized, though more weakly, for $P$ wave incidence on the horizontal cracks (Figs. 10(b) and 10(c)). Such a feature was previously reported in Paper I on $S H$ wave scattering in the case of an even higher crack density $(\epsilon=0.2)$ (Fig. 12 thereof). As discussed therein, this may be partly attributed to the effect of multiple scattering among closely located scatterers. The FAT is based on the randomness and sparseness of scatterer distributions as stated in Section 1. Speaking precisely, however, a distribution of non-overlapping scatterers cannot be perfectly random for a finite distribution density. In the present case, with such dense distributions, the randomness would be largely degraded, which results in large approximation errors. Note that a more rigorous treatment of mean waves revealed that the terms of order $\epsilon^{2}$, neglected in the FAT of Kawahara (1992), can be described in terms of such nonrandomness (Keller, 1964). Only in the case of $P$ wave incidence on the horizontal cracks, may one also perceive the first motion of the predicted mean wave prior to the onset of the initial wavelet without cracks (at around $t=7.3$ ), which is not observed for the synthetic case (Fig. 10(a)). Such apparently acausal wave motion was again reported in Paper I in the case of $\epsilon=0.2$, which we attributed to a too strong attenuation $\left(Q^{-1} \sim 1\right)$. Throughout the present paper, as well as Paper I, we have assumed the exponential decay expressed by $\exp \left(-k Q^{-1} x / 2\right)$ as usual, with $x$ being the travel distance. 
Parallel P wave incidence, $\varepsilon=0.0125$ (Model 1)
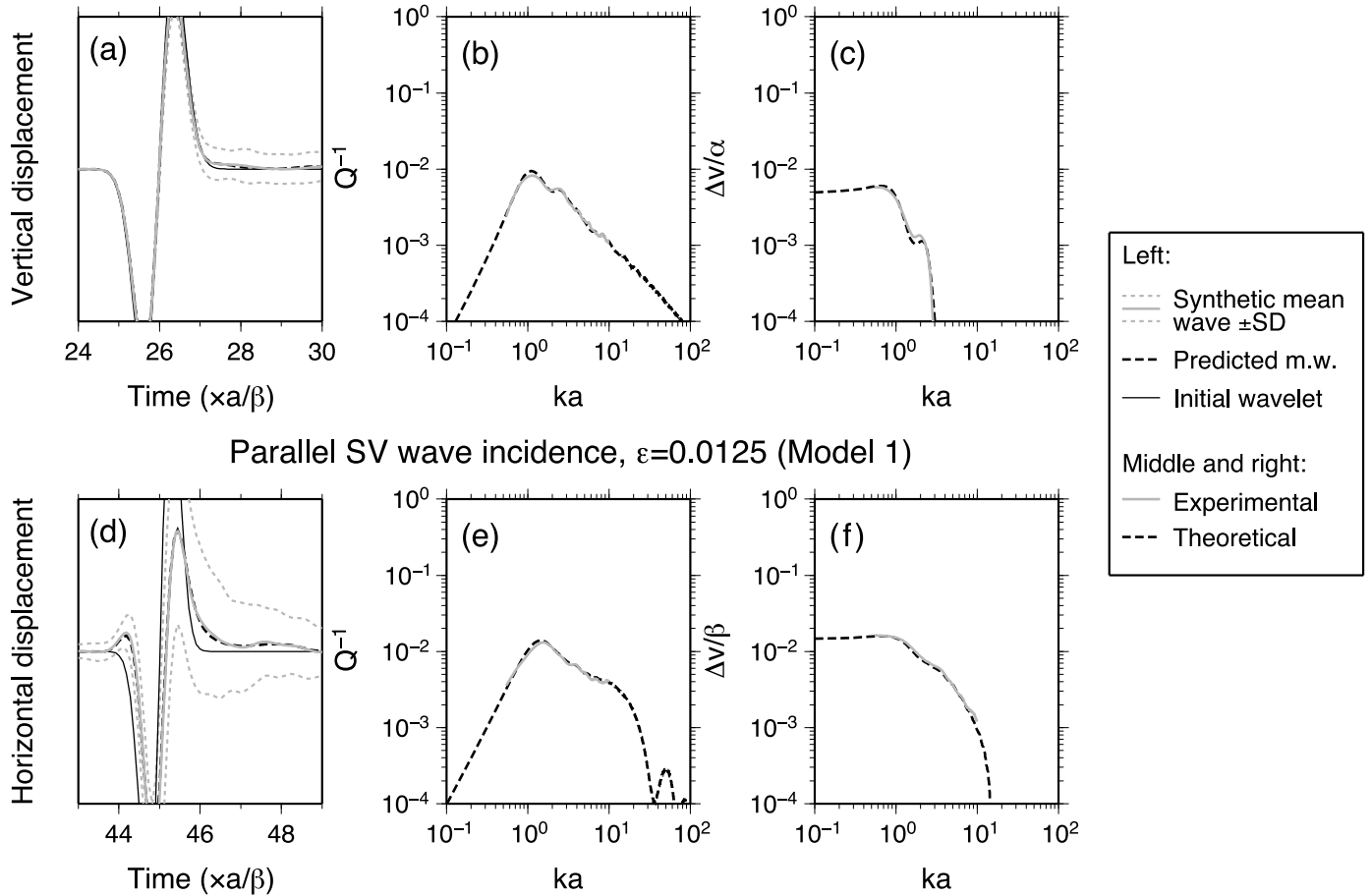

Fig. 8. Results of the simulations obtained for Model $1(\epsilon=0.0125)$ with either $(a-c) P$ or $(d-f) S V$ wave incidence on vertical cracks. Left column $(\mathrm{a}, \mathrm{d})$ : Synthetic mean waves and their standard deviation derived from synthetic seismograms (solid and dotted gray curves, respectively) of either (a) vertical- or (d) horizontal-component. The dashed black curves denote the predicted mean waves, and the thin solid black ones denote the initial wavelets, if without the cracks. Middle (b, e) and right (c, f) columns: $Q^{-1}$ and $\Delta v$ for the synthetic mean waves (solid gray curves), respectively. The prediction by the FAT is denoted by dashed black curves.

Strictly, however, it is an approximation valid for weak attenuation, i.e., $Q^{-1} \ll 1$ (e.g., Aki and Richards, 2002). This is obviously not satisfied in the present case, in which the maximum value of $Q^{-1}$ is rather close to 1 , probably making both the theory and measurement not very reliable.

In contrast, the disagreement between the experimental and theoretical results still remains not very serious for $S V$ wave incidence, even in the case of $\epsilon=0.1$ (Figs. 10(d) to $10(\mathrm{f})$ and $10(\mathrm{j})$ to $10(\mathrm{l})$ ). One may therefore say that the FAT will be nearly valid at least up to $\epsilon=0.1$ concerning $S V$ waves. This is consistent with the conclusion of Paper I as well as that of Murai (2007) on $S H$ wave scattering.

We notice that the case of $P$ wave incidence on vertical cracks with $\epsilon=0.1$ was examined on the basis of Model $3 \mathrm{~b}$ with $N=152$, many more cracks than assumed in Model 3a adopted for the other three cases, for the same reason as before (Model 2). In Paper I, however, we revealed that the numerical errors of the FDM seem to weakly increase with an increasing number of cracks. Hence, one might infer that the failure of the FAT, in that case, is partly because of the numerical errors. To examine this inference, we recalculate $Q^{-1}$ and $\Delta v$ for this case by replacing Model 3b with Model 3a. The result (not shown) is effectively unchanged from that depicted in Figs. 10(h) and 10(i), indicating that the FDM calculations were sufficiently accurate and the failure of the FAT, in this case, is not due to the numerical errors caused by too many cracks.

One may also infer that strong multiple scattering among the cracks enhances the numerical errors. This effect would be significant for a high crack density and especially for high wavenumbers, even if the sampling criterion (Section 2) is satisfied. However, we confirmed that changing $\Delta h$ by a factor of two does not effectively change the numerical results for Model $3 \mathrm{a}$ for all the considered wavenumbers (figure omitted). This again verifies the accuracy of the present FDM simulations, even up to $\epsilon=0.1$ and the considered wavenumbers.

In summary, we have confirmed that the FAT is actually valid for low crack density (say, $\epsilon \sim 0.01$ ) for all the cases considered. Its validity limit with regard to $\epsilon$, however, seems to depend on the wave modes and, sometimes, the incident angles of waves to cracks. The FAT would be nearly valid with $\epsilon$ up to at least 0.1 for the case of $S V$ wave incidence. In contrast, the validity limit of the FAT seems to be lower for $P$ wave incidence. It may be, say, 0.05 for normal incidence, whereas even a lower value of $\epsilon$ may be preferable for a grazing incidence.

\section{Discussion and Conclusions}

In this paper, we have implemented a simple, but effective, finite-difference scheme to simulate $P-S V$ wave scattering due to 2-D parallel cracks. We expressed tractionfree, infinitesimally thin cracks in a finite-difference staggered grid in probably the simplest manner: we assumed arrays of grid points with zero shear traction. We split the grid points for vertical particle velocity on the planes into pairs of very close points, thus succeeding in expressing the NDD along the planes. As an accuracy test, we calculated the displacement discontinuity along an isolated crack acted on by harmonic waves using the present method and com- 
Normal $\mathrm{P}$ wave incidence, $\varepsilon=0.05$ (Model 2a)
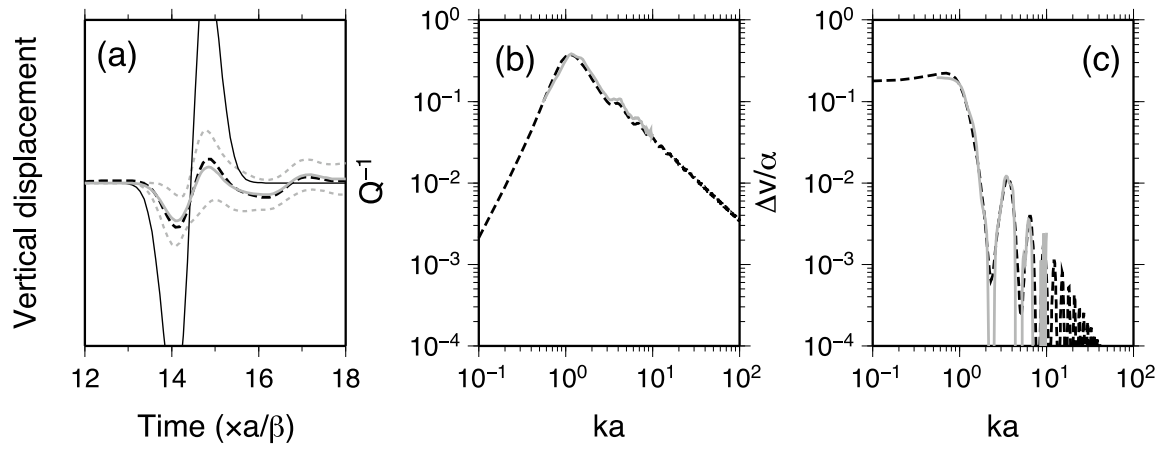

Normal SV wave incidence, $\varepsilon=0.05$ (Model 2a)
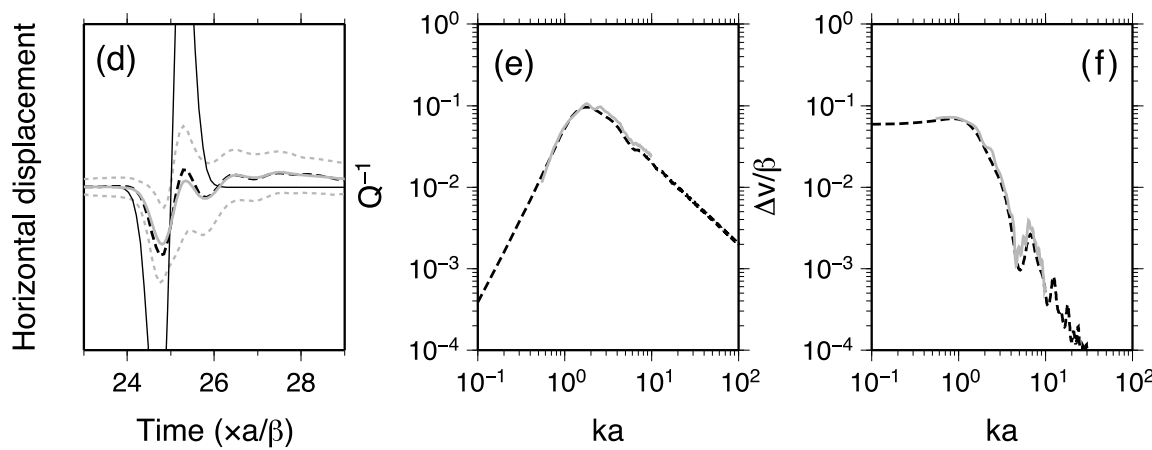

Parallel $P$ wave incidence, $\varepsilon=0.05$ (Model 2b)
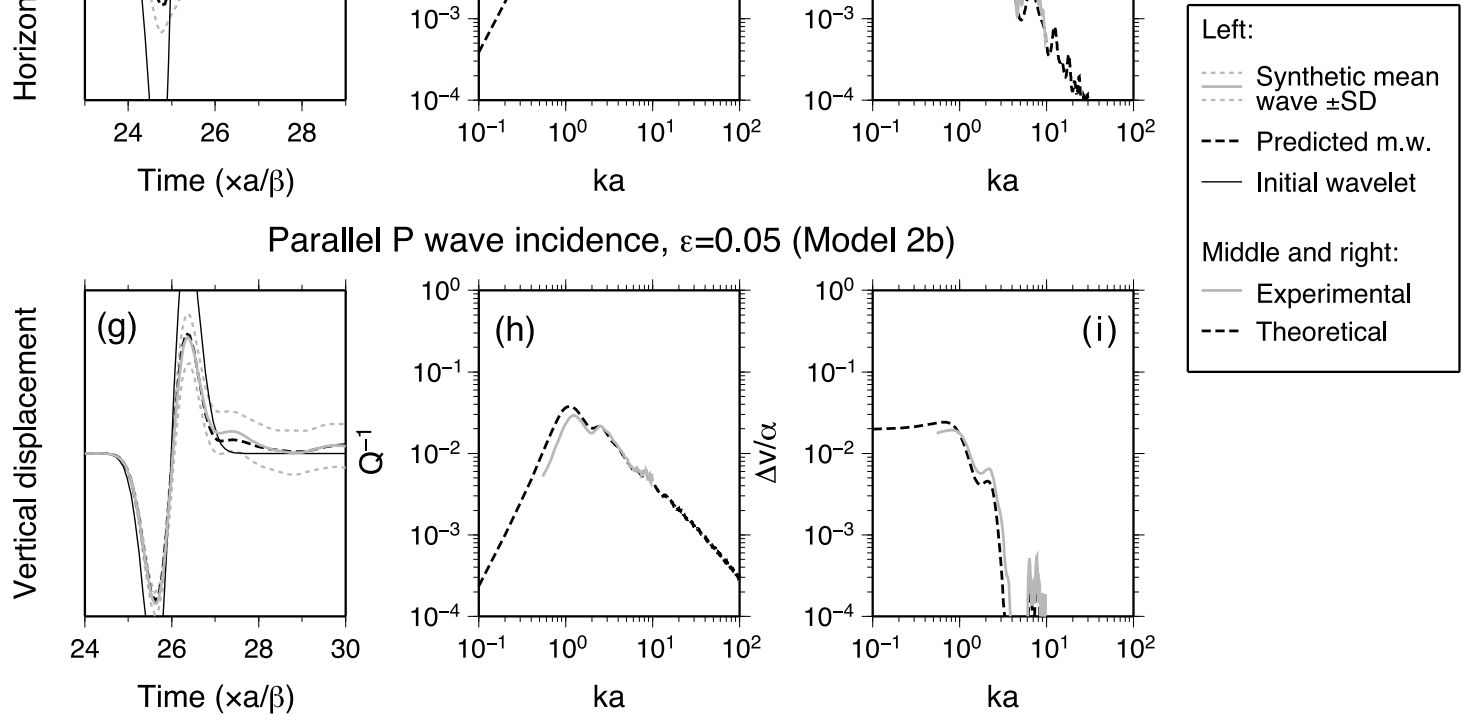

Parallel SV wave incidence, $\varepsilon=0.05$ (Model 2a)
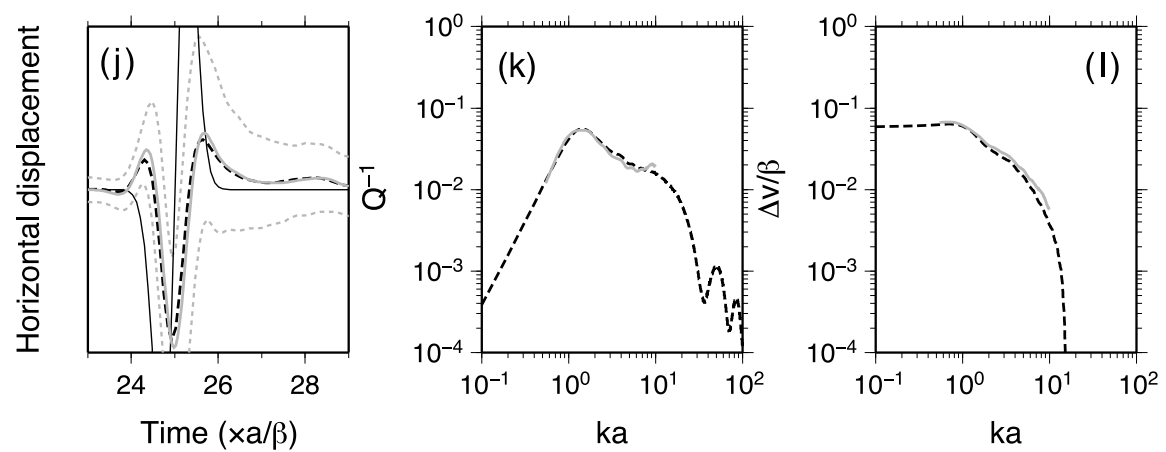

Fig. 9. Results of the simulations obtained for Model $2(\epsilon=0.05)$ with either $(\mathrm{a}-\mathrm{c}) P$ or $(\mathrm{d}-\mathrm{f}) S V$ wave incidence on horizontal cracks, and those with either (g-i) $P$ or (j-1) $S V$ wave incidence on vertical cracks, shown in the same manner as in Fig. 8. Left column (a, d, g, j): Synthetic mean waves and their standard deviation derived from synthetic seismograms of either (a, g) vertical- or (d, j) horizontal-component. The predicted mean waves and the initial wavelets, if without the cracks, are also depicted. Middle (b, e, h, k) and right (c, f, i, l) columns: $Q^{-1}$ and $\Delta v$ for the synthetic mean waves, respectively.

pared this with the corresponding results based on a reliable BIEM of Kawahara and Yamashita (1992). The test resultantly indicated that the present method yielded a sufficient accuracy for any wavenumber. As an application of the method, we next performed simulations of wave propagation in media with randomly distributed parallel cracks of the same length. We determined experimentally the attenuation and velocity dispersion induced by scattering from the 
Normal $P$ wave incidence, $\varepsilon=0.1$ (Model 3a)
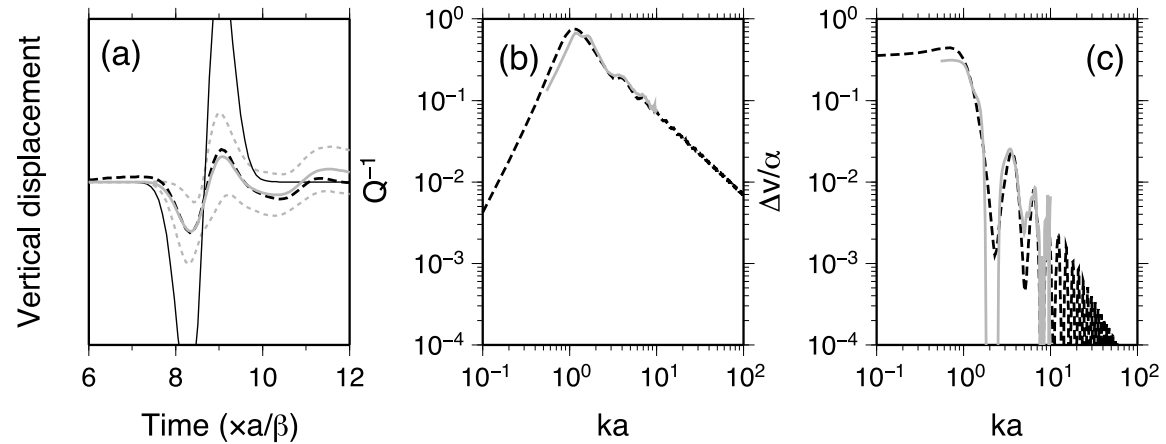

Normal SV wave incidence, $\varepsilon=0.1$ (Model 3a)
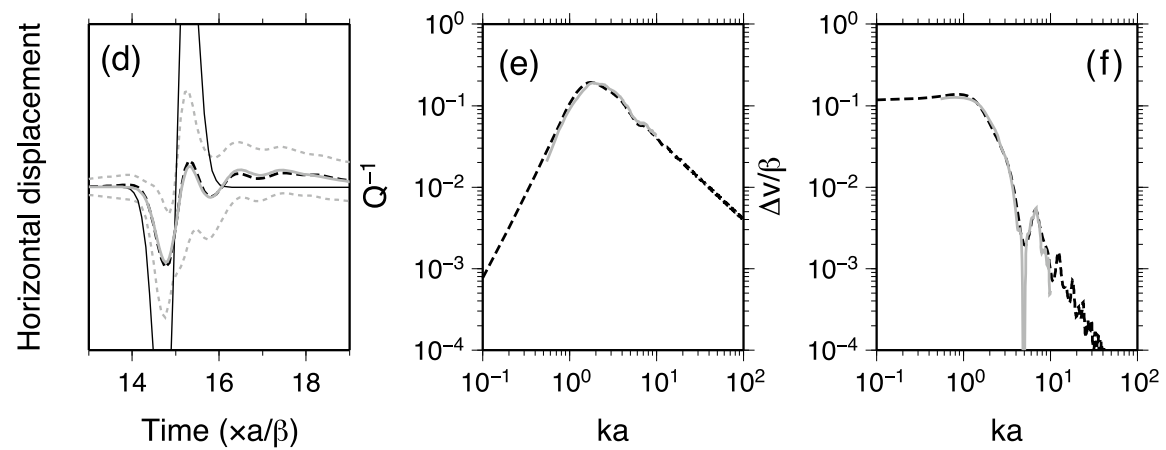

Parallel $\mathrm{P}$ wave incidence, $\varepsilon=0.1$ (Model $3 b$ )
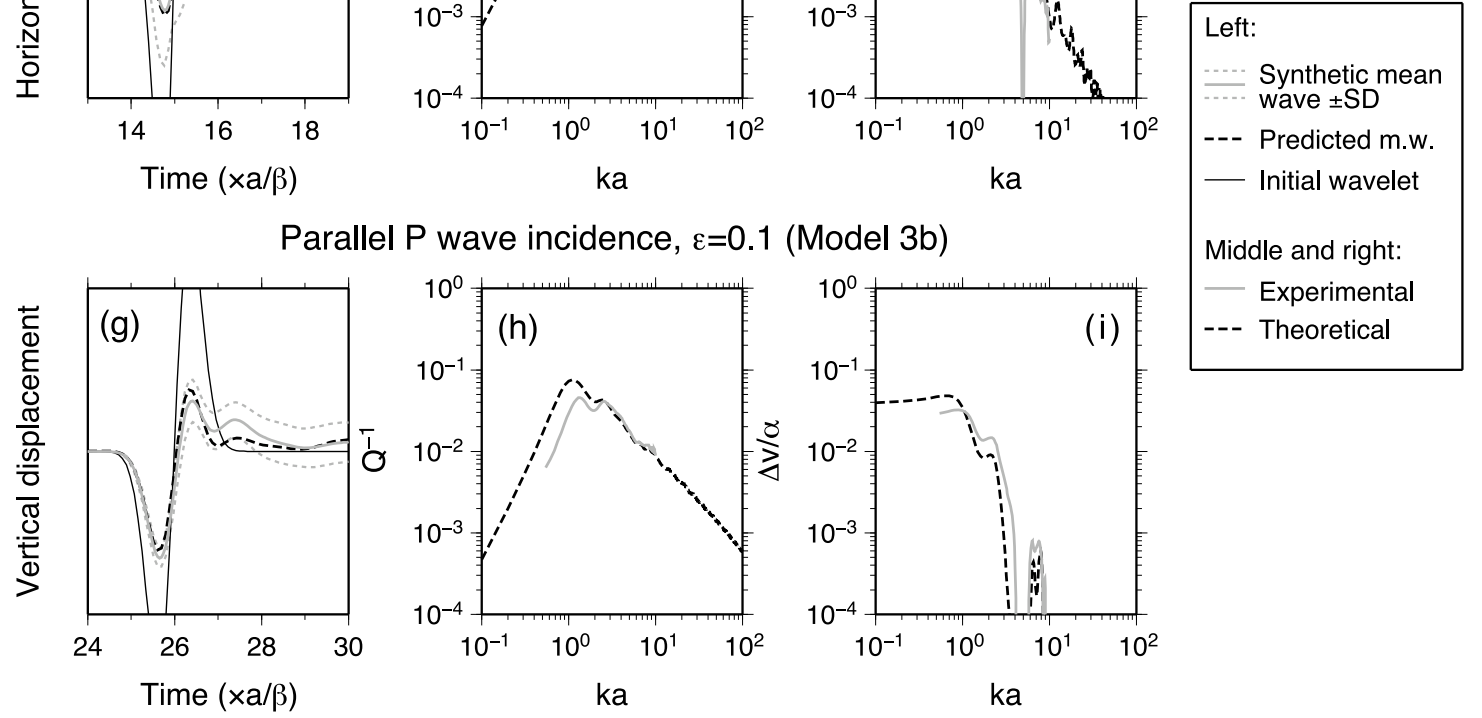

Parallel SV wave incidence, $\varepsilon=0.1$ (Model 3a)
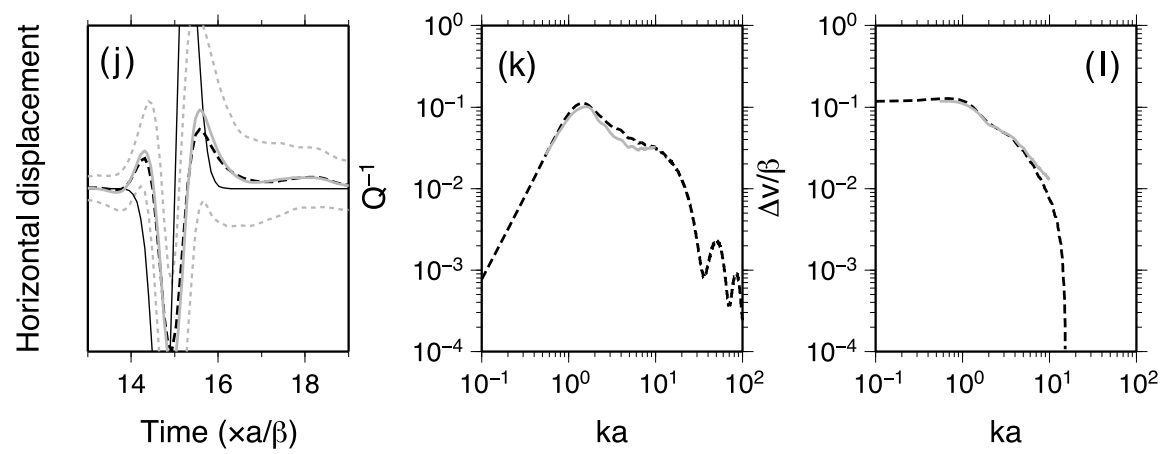

Fig. 10. Results of the simulations obtained for Model $3(\epsilon=0.1)$ with either $(\mathrm{a}-\mathrm{c}) P$ or $(\mathrm{d}-\mathrm{f}) S V$ wave incidence on horizontal cracks, and those with either (g-i) $P$ or (j-1) $S V$ wave incidence on vertical cracks, shown in the same manner as in Fig. 9. Left column (a, d, g, j): Synthetic mean waves and their standard deviation derived from synthetic seismograms of either (a, g) vertical- or (d, j) horizontal-component. The predicted mean waves and the initial wavelets, if without the cracks, are also depicted. Middle (b,e, h, k) and right $(\mathrm{c}, \mathrm{f}, \mathrm{i}, \mathrm{l})$ columns: $Q^{-1}$ and $\Delta v$ for the synthetic mean waves, respectively.

synthetic seismograms, using a waveform averaging technique. It was shown that the results are well described by the FAT of Kawahara (1992), if the crack density $\epsilon$ is sufficiently low, say, 0.01 . The FAT apparently remains to be always valid with $\epsilon$ up to at least 0.1 for $S V$ wave incidence, whereas the validity limit seems to be lower for $P$ wave incidence; it may be, say, 0.05 for normal incidence and even lower for grazing incidence. 
To the authors' knowledge, the present study is the first one to have successfully validated the FAT for cracked media, even if there exists mode conversion between $P$ and $S$ waves. Although we only dealt with the simplest type of cracks (i.e., parallel equal-sized traction-free cracks), some previous studies based similarly on numerical experiments showed that the FAT works well also for others kinds of cracks concerning $S H$ wave scattering. The examples are cracks filled with a viscous fluid (Murai et al., 1995), randomly oriented cracks (Yoshida et al., 2003), and cracks of unequal lengths (Paper I). On the basis of the same approach as the present study, Kawahara et al. (2009) also proved that the FAT is nearly valid for $S H$ wave scattering due to 2-D round cavities-possibly identified with high-aspect-ratio cracks - with a volume concentration up to 0.1 . All these results suggests that the validity of the FAT would be universal as long as the scatterers are sparsely distributed, irrespective of the geometry and physical properties (boundary conditions) of the cracks, the wave modes, and, possibly, the spatial dimensions.

In contrast, the validity limit of the FAT for cracked media seems to depend on the wave modes. Moreover, this is as well as the incident angle in the case of $P$ wave incidence. The details of the dependence of the validity limit on the incident angle, however, remain unrevealed since we considered here only two kinds of incident angles $\left(0^{\circ}\right.$ and $90^{\circ}$ ); the case of oblique wave incidence should be investigated in future. It also remains to be clarified why the validity limit of the FAT seems to be lower for $P$ wave incidence than for $S V$ wave incidence.

As stated in Section 4, the overestimation by the FAT of $Q^{-1}$ and $\Delta v$ observed for $P$ waves in densely cracked media (Fig. 10) may be partly attributed to the imperfect randomness of distributions enhanced with an increasing $\epsilon$. This implies that the validity of the FAT would be limited not only by $\epsilon$ but also by the spatial correlation among cracks. Murai (2007) demonstrated the importance of the spatial correlation by evaluating $Q^{-1}$ and $\Delta v$ of $S H$ waves in media with periodically arrayed cracks, and with $\epsilon=$ 0.1 , through numerical experiments. The obtained $Q^{-1}$ and $\Delta v$ were compared with those for (nearly) random crack distributions with the same $\epsilon$. It was revealed that the periodicity (or strong correlation) of crack locations largely reduces $Q^{-1}$ for $k a<1$ and caused the FAT to fail. This result is qualitatively consistent with the present results. However, his result also indicated that the periodicity hardly affects $\Delta v$, in contrast to the present result that $\Delta v$ is also reduced for $k a<1$ with increasing $\epsilon$. Note that Murai adopted a peak picking technique in evaluating $Q^{-1}$ and $\Delta v$, unlike us; how the choice of the evaluation methods affects the results would be of interest. Future works should also reveal the effect of the crack spatial correlation on $Q^{-1}$ and $\Delta v$ concerning $P-S V$ wave scattering.

We notice that Orlowsky et al. (2003) numerically evaluated the effective velocity (phase velocity in the longwavelength limit), $v_{\text {eff }}$, of elastic waves normally impinging on 2-D parallel empty cracks. Their results indicate that $v_{\text {eff }}$ of $P$ waves for $\epsilon=0.1$ is, in our notation, about $0.7 \alpha$ (i.e., $\Delta v / \alpha \approx 0.3$ ) and that of $S V$ waves is about $0.87 \beta$ (i.e., $\Delta v / \beta \approx 0.13$ ). These values are roughly consistent with the theoretical values of $\Delta v$ at $k a=0.1$ for the same $\epsilon$ shown in Figs. 10(c) and 10(f). Unfortunately, a comparison of the experimental values shown in these figures with $v_{\text {eff }}$ by Orlowsky et al. (2003) may not be straightforward because the former were measured only for $k a>0.5$. Figure 10(c, i, f, 1), as well as Kawahara (1992), also suggests that a medium with parallel empty cracks is strongly anisotropic for $P$ waves but nearly isotropic for $S V$ waves; for example, Fig. 10 shows that the theoretical value of $\Delta v / \alpha$ at $k a=0.1$ for $P$ waves for normal incidence is nearly ten times larger than that for parallel incidence. Note also that Saenger and Shapiro (2002) estimated $v_{\text {eff }}$ in media with 2-D randomly orientated empty cracks. Comparison of their results with ours implies that $v_{\text {eff }}$ of $P$ waves for randomly oriented cracks lies between those for a normal and parallel incidence on parallel cracks, whereas $v_{\text {eff }}$ of $S$ waves is nearly independent of the crack orientations. These features are shared by media with pennyshaped empty cracks (Crampin, 1984).

Although the simplicity of the present FDM is a great advantage, this method, as it stands, has some limitations on the geometry of cracks. One of these is that cracks must be parallel to the grid lines so that one can only choose, for 2-D grids, one or two orientations of cracks simultaneously. For a wider application of the present FDM (e.g., a medium with randomly oriented cracks), it is crucial to be able to incorporate arbitrarily oriented cracks into the standard staggered grid used in the present FDM. We infer that this will be achieved on the basis of the staircase approximation as has been successfully applied to irregular free surfaces and irregular liquid-solid boundaries (Ohminato and Chouet, 1997; Okamoto and Takenaka, 2005; Takenaka et $a l ., 2009)$. It is worthwhile stating, however, that there are no limitations, even in the present FDM, on the incident angle of elastic waves to a crack, although we considered here only two incident angles. For example, one could simulate scattering by parallel cracks of a plane wave with an oblique incidence if the source is appropriately given in the grid.

Another limitation of the present FDM may be that it can treat non-intersecting empty cracks only. Relaxation of these limitations should be attempted in future works. A recent development has been achieved by Shiina et al. (2011), who succeeded in modeling 2-D cracks filled with a viscous fluid in the standard staggered grid and simulated the consequent scattering of $S H$ waves with high accuracy. Extension of their method to $P-S V$ wave scattering would be of value.

Acknowledgments. The authors would like to thank Satoshi Nozawa and the members of his laboratory for using their computing environment and fruitful discussion. The critical comments of Marcel Frehner and an anonymous reviewer helped to largely improve the manuscript. This study was partly supported by the Earthquake Research Institute Cooperative Research Program (2000-B-07, 2003-B-04). All the figures in this paper were generated using the GMT software of Wessel and Smith (1998).

\section{Appendix A. Derivation of Eq. (2)}

We start with the equation of motion for $P-S V$ waves. An equation for the vertical component of acceleration $\partial v_{z} / \partial t$ 
is given in the form:

$$
\rho \partial_{t} v_{z}=\partial_{x} \tau_{x z}+\partial_{z} \tau_{z z}
$$

in which $\partial_{t}=\partial / \partial t, \partial_{x}=\partial / \partial x$, etc. Now let us introduce the staggered grid shown in Fig. 1, and consider the finite difference approximation of the above equation for a grid point $(i, j+\delta)$ (a point on the lower crack face) and time $t=$ $m \Delta t$. A second-order central difference formula (Moczo, 1998) for $\partial_{t} v_{z}$ in the form

$$
\begin{aligned}
& \partial_{t} v_{z}(i, j+\delta, m) \approx(\Delta t)^{-1} \\
& \quad \times\left[v_{z}\left(i, j+\delta, m+\frac{1}{2}\right)-v_{z}\left(i, j+\delta, m-\frac{1}{2}\right)\right],
\end{aligned}
$$

which has been used in Eq. (1b), is applicable also in this case. Additionally,

$$
\partial_{x} \tau_{x z}(i, j+\delta, m)=0
$$

because of the stress-free boundary condition on the crack face $\left(\tau_{x z}=0\right)$. However, the central difference formula for $\partial_{z} \tau_{z z}$ used in Eq. (1b) is inapplicable in the present case because it requires the value of $\tau_{z z}$ at the point $\left(i, j-\frac{1}{2}\right)$ across the crack, which would cause errors (see subsection 3.1 of Paper I). We instead adopt a forward difference approximation in the following manner. Performing a Taylor series expansion of $\tau_{z z}$ around a point with $z=z_{0}$ in Cartesian coordinates, we obtain:

$$
\begin{aligned}
&\left.\tau_{z z}\right|_{z=z_{0}}+\frac{1}{2} \Delta h=\left.\tau_{z z}\right|_{z=z_{0}}+\left.\partial_{z} \tau_{z z}\right|_{z=z_{0}} \frac{1}{2} \Delta h \\
&+\left.\frac{1}{2} \partial_{z z} \tau_{z z}\right|_{z=z_{0}}\left(\frac{1}{2} \Delta h\right)^{2}+O\left(\Delta h^{3}\right), \\
&\left.\tau_{z z}\right|_{z=z_{0}}+\frac{3}{2} \Delta h=\left.\tau_{z z}\right|_{z=z_{0}}+\left.\partial_{z} \tau_{z z}\right|_{z=z_{0}} \frac{3}{2} \Delta h \\
&+\left.\frac{1}{2} \partial_{z z} \tau_{z z}\right|_{z=z_{0}}\left(\frac{3}{2} \Delta h\right)^{2}+O\left(\Delta h^{3}\right) .
\end{aligned}
$$

From these two equations, we get:

$$
\begin{aligned}
\left.\partial_{z} \tau_{z z}\right|_{z=z_{0}}= & \frac{1}{3 \Delta h}\left[-\left.8 \tau_{z z}\right|_{z=z_{0}}+\left.9 \tau_{z z}\right|_{z=z_{0}+\frac{1}{2} \Delta h}\right. \\
& \left.-\left.\tau_{z z}\right|_{z=z_{0}+\frac{3}{2} \Delta h}\right]+O\left(\Delta h^{2}\right) .
\end{aligned}
$$

Now let the present point coincide with the grid point $(i, j+$ $\delta)$. Although $\tau_{z z}$ is not allocated on this grid point (Fig. 1), it does not matter because we can set $\left.\tau_{z z}\right|_{z=z_{0}}$ in Eq. (A.5) to be 0 due to the stress-free boundary condition on the crack face. We thus obtain the following approximation:

$$
\begin{aligned}
\partial_{z} \tau_{z z}(i, j+\delta, m) \approx(3 \Delta h)^{-1} & \\
& \times\left[9 \tau_{z z}\left(i, j+\frac{1}{2}, m\right)-\tau_{z z}\left(i, j+\frac{3}{2}, m\right)\right] .
\end{aligned}
$$

This is a second-order forward difference formula, which satisfies the boundary condition on the crack face and requires no quantities at points across the crack. Note that an equivalent formula was previously obtained by Hayashida et al. (1999) (their equation (11)). Substituting Eqs. (A.2), (A.3) and (A.6) into Eq. (A.1) and rewriting $v_{z}(i, j+\delta, m \pm$ $\left.\frac{1}{2}\right)=v_{z}^{+}\left(i, j, m \pm \frac{1}{2}\right)$, we finally obtain Eq. (2) with the superscript "+". Equation (2) with the superscript "_," can be also obtained for the upper crack face in the same manner.

\section{References}

Aki, K. and P. G. Richards, Quantitative Seismology, 2nd edition, 699 pp., University Science Books, Sausalito, California, 2002.

Benites, R., K. Aki, and K. Yomogida, Multiple scattering of SH waves in 2-D media with many cavities, Pure Appl. Geophys., 138, 353-390, 1992.

Caleap, M. and C. Aristégui, Effective antiplane properties in the presence of frictional shear cracks, J. Geophys. Res., 115, B02302, doi:10.1029/2009JB006485, 2010.

Clayton, R. and B. Engquist, Absorbing boundary conditions for acoustic and elastic equations, Bull. Seismol. Soc. Am., 67, 1529-1540, 1977.

Crampin, S., Effective anisotropic elastic constants for wave propagation through cracked solids, Geophys. J. R. Astron. Soc., 76, 135-145, 1984.

Dahm, T. and T. Becker, On the elastic and viscous properties of media containing strongly interacting in-plane cracks, Pure Appl. Geophys., 151, 1-16, 1998.

Foldy, L. L., The multiple scattering of waves. I. General theory of isotropic scattering by randomly distributed scatterers, Phys. Rev., 67, 107-119, 1945.

Frehner, M. and S. M. Schmalholz, Finite-element simulations of Stoneley guided-wave reflection and scattering at the tips of fluid-filled fractures, Geophysics, 75, T23-T36, 2010.

Frehner, M., S. M. Schmalholz, E. H. Saenger, and H. Steeb, Comparison of finite difference and finite element methods for simulating twodimensional scattering of elastic waves, Phys. Earth Planet. Inter., 171, 112-121, 2008.

Grechka, V., Comparison of the non-interacting and differential schemes in predicting the effective elastic properties of fractured media, Int. J. Fract., 144, 181-188, 2007.

Grechka, V. and M. Kachanov, Effective elasticity of rocks with closely spaced and intersecting cracks, Geophysics, 71, D85-D91, 2006.

Hayashida, T., H. Takenaka, and T. Okamoto, Development of 2D and $3 \mathrm{D}$ codes of the velocity-stress staggered-grid finite-difference method for modeling seismic wave propagation, Sci. Repts., Dept. Earth \& Planet. Sci., Kyushu Univ., 20, 99-110, 1999 (in Japanese with English abstract).

Ishimaru, A., Wave Propagation and Scattering in Random Media, Vols. 1 and 2, 609pp., Academic Press, New York, 1978 (reissued in 1997 by IEEE Press and Oxford Univ. Press, New York).

Kawahara, J., Scattering of P, SV waves by a random distribution of aligned open cracks, J. Phys. Earth, 40, 517-524, 1992.

Kawahara, J., Scattering attenuation of elastic waves due to low-contrast inclusions, Wave Motion, 48, 290-300, 2011.

Kawahara, J. and T. Yamashita, Scattering of elastic waves by a fracture zone containing randomly distributed cracks, Pure Appl. Geophys., 139, 121-144, 1992.

Kawahara, J., T. Ohno, and K. Yomogida, Attenuation and dispersion of antiplane shear waves due to scattering by many two-dimensional cavities, J. Acoust. Soc. Am., 125, 3589-3596, 2009.

Keller, J. B., Stochastic equations and wave propagation in random media, Proc. Symp. Appl. Math., 16, 145-170, 1964.

Kelner, S., M. Bouchon, and O. Coutant, Numerical simulation of the propagation of $\mathrm{P}$ waves in fractured media, Geophys. J. Int., 137, $197-$ 206, 1999.

Kikuchi, M., Dispersion and attenuation of elastic waves due to multiple scattering from inclusions, Phys. Earth Planet. Inter., 25, 159-162, 1981a.

Kikuchi, M., Dispersion and attenuation of elastic waves due to multiple scattering from cracks, Phys. Earth Planet. Inter., 27, 100-105, 1981b.

Krüger, O. S., E. H. Saenger, and S. A. Shapiro, Scattering and diffraction by a single crack: an accuracy analysis of the rotated staggered grid, Geophys. J. Int., 162, 25-31, 2005.

Levander, A. R., Fourth-order finite-difference P-SV seismograms, Geophysics, 53, 1425-1436, 1988.

Liu, E. and Z. Zhang, Numerical study of elastic wave scattering by cracks or inclusions using the boundary integral equation method, J. Comp. Acoust., 9, 1039-1054, 2001.

Madariaga, R., Dynamics of an expanding circular fault, Bull. Seismol. Soc. Am., 66, 639-666, 1976.

Mal, A. K., Interaction of elastic waves with a Griffith crack, Int. J. Eng Sci., 8, 763-776, 1970.

Moczo, P., Introduction to Modeling Seismic Wave Propagation by the Finite-difference Method, 102 pp., Lecture Notes, Disaster Prevention Research Institute, Kyoto University, 1998.

Moczo, P., J. Kristek, V. Vavryčuk, R. J. Archuleta, and L. Halada, 3D het- 
erogeneous staggered-grid finite-difference modeling of seismic motion with volume harmonic and arithmetic averaging of elastic moduli and densities, Bull. Seismol. Soc. Am., 92, 3042-3066, 2002.

Murai, Y., Scattering attenuation, dispersion and reflection of $\mathrm{SH}$ waves in two-dimensional elastic media with densely distributed cracks, Geophys. J. Int., 168, 211-223, 2007.

Murai, Y., J. Kawahara, and T. Yamashita, Multiple scattering of SH waves in 2-D elastic media with distributed cracks, Geophys. J. Int., 122, 925937, 1995.

Nakamura, T., H. Takenaka, T. Okamoto, and Y. Kaneda, FDM simulation of seismic-wave propagation for an aftershock of the 2009 Suruga Bay earthquake: effects of ocean-bottom topography and seawater layer, Bull. Seismol. Soc. Am., 102, 2420-2435, doi:10.1785/0120110356, 2012.

Ohminato, T. and B. A. Chouet, A free-surface boundary condition for including 3D topography in the finite-difference method, Bull. Seismol. Soc. Am., 87, 494-515, 1997.

Okamoto, T. and H. Takenaka, Fluid-solid boundary implementation in the velocity-stress finite-difference method, Zisin (J. Seismol. Soc.Jpn.), 57, 355-364, 2005 (in Japanese with English abstract).

Okamoto, T., H. Takenaka, T. Nakamura, and T. Aoki, Large-scale simulation of seismic-wave propagation of the 2011 Tohoku-Oki M9 earthquake, Proc. Int. Symp. on Engineering Lessons Learned from the 2011 Great East Japan Earthquake, 349-360, Tokyo, Japan, 2012.

Okamoto, T., H. Takenaka, T. Nakamura, and T. Aoki, Accelerating largescale simulation of seismic wave propagation by multi-GPUs and threedimensional domain decomposition, in GPU Solutions to Multi-scale Problems in Science and Engineering, edited by D. A. Yuen et al., Chap. 24, 375-389, doi:10.1007/978-3-642-16405-7_24, Springer Berlin Heidelberg, 2013.

Orlowsky, B., E. H. Saenger, Y. Guéguen, and S. A. Shapiro, Effects of parallel crack distributions on effective elastic properties-a numerical study, Int. J. Fract., 124, L171-L178, 2003.

Pointer, T., E. Liu, and J. A. Hudson, Numerical modelling of seismic waves scattered by hydrofractures: application of the indirect boundary element method, Geophys. J. Int., 135, 289-303, 1998.

Saenger, E. H. and S. A. Shapiro, Effective velocities in fractured media: a numerical study using the rotated staggered finite-difference grid, Geophys. Prospect., 50, 183-194, 2002.

Saenger, E. H., N. Gold, and S. A. Shapiro, Modeling the propagation of elastic waves using a modified finite-difference grid, Wave Motion, 31, 77-79, 2000.

Saenger, E. H., O. S. Krüger, and S. A. Shapiro, Modeling the propagation of elastic waves using a modified finite-difference grid, Geophys. Prospect., 52, 183-195, 2004.

Saenger, E. H., O. S. Krüger, and S. A. Shapiro, Effective elastic properties of fractured rocks: dynamic vs. static considerations, Int. J. Fract., 139, 569-576, 2006.

Sato, H., M. C. Fehler, and T. Maeda, Seismic Wave Propagation and Scattering in the Heterogeneous Earth, 2nd ed., 494 pp., Springer Berlin Heidelberg, 2012.

Shapiro, S. A. and G. Kneib, Seismic attenuation by scattering: theory and numerical results, Geophys. J. Int., 114, 373-391, 1993.

Shiina, T., J. Kawahara, and T. Okamoto, 2-D finite difference simulations of SH waves scattered by cracks filled with viscous fluid, Prog. Abstr. Seismol. Soc. Jpn. 2011 Fall Meet., D21-09, 2011 (in Japanese).

Suzuki, Y., J. Kawahara, T. Okamoto, and K. Miyashita, Simulations of SH wave scattering due to cracks by the 2-D finite difference method, Earth Planets Space, 58, 555-567, 2006.

Takenaka, H., T. Nakamura, T. Okamoto, and Y. Kaneda, A unified approach implementing land and ocean-bottom topographies in the staggered-grid finite-difference method for seismic wave modeling, Proc. 9th SEGJ Int. Symp., Sapporo, Japan, 1-4, doi: 10.1190/segj092009-001.13, 2009.

Van Antwerpen, V. A., W. A. Mulder, and G. C. Herman, Finite-difference modeling of two-dimensional elastic wave propagation in cracked media, Geophys. J. Int., 149, 169-178, 2002.

Virieux, J., SH-wave propagation in heterogeneous media: velocity-stress finite-difference method, Geophysics, 49, 1933-1957, 1984.

Virieux, J., P-SV wave propagation in heterogeneous media: velocitystress finite-difference method, Geophysics, 51, 889-901, 1986.

Vlastos, S., E. Liu, I. G. Main, and X.-Y. Li, Numerical simulation of wave propagation in media with discrete distributions of fractures: effects of fracture sizes and spatial distributions, Geophys. J. Int., 152, 649-668, 2003.

Wessel, P. and W. H. F. Smith, New, improved version of the Generic Mapping Tools Released, Eos Trans. AGU, 79, 579, 1998.

Yamashita, T., Attenuation and dispersion of $\mathrm{SH}$ waves due to scattering by randomly distributed cracks, Pure Appl. Geophys., 132, 545-568, 1990.

Yomogida, K. and R. Benites, Scattering of seismic waves by cracks with the boundary integral method, Pure Appl. Geophys., 159, 1771-1789, 2002.

Yoshida, T., J. Kawahara, and Y. Murai, Simulations of SH waves scattered by randomly oriented 2-D cracks, Abstr. 2003 Jpn. Earth Planet. Sci. Joint Meet., S047-P007, 2003.

Zhang, Ch. and D. Gross, On Wave Propagation in Elastic Solids with Cracks, 248 pp., Computational Mechanics, Southampton, UK, 1997.

Y. Suzuki, T. Shiina, J. Kawahara (e-mail: junk@mx.ibaraki.ac.jp), T. Okamoto, and K. Miyashita 